\title{
Optimal operation of integrated electricity and heat system: A review of modeling and
} solution methods

\author{
Zhang, Menglin; Wu, Qiuwei; Wen, Jinyu; Lin, Zhongwei; Fang, Fang; Chen, Qun
}

\section{Published in:}

Renewable \& Sustainable Energy Reviews

Link to article, DOI:

10.1016/j.rser.2020.110098

Publication date:

2020

Document Version

Peer reviewed version

Link back to DTU Orbit

Citation (APA):

Zhang, M., Wu, Q., Wen, J., Lin, Z., Fang, F., \& Chen, Q. (2020). Optimal operation of integrated electricity and heat system: A review of modeling and solution methods. Renewable \& Sustainable Energy Reviews, [110098]. https://doi.org/10.1016/j.rser.2020.110098

\section{General rights}

Copyright and moral rights for the publications made accessible in the public portal are retained by the authors and/or other copyright owners and it is a condition of accessing publications that users recognise and abide by the legal requirements associated with these rights.

- Users may download and print one copy of any publication from the public portal for the purpose of private study or research.

- You may not further distribute the material or use it for any profit-making activity or commercial gain

- You may freely distribute the URL identifying the publication in the public portal 


\section{Optimal operation of integrated electricity and heat system: A review of}

\section{2 modeling and solution methods}

3 Menglin Zhang ${ }^{\mathrm{a}}$, Qiuwei Wu ${ }^{\mathrm{a}}$, , Jinyu Wen ${ }^{\mathrm{b}}$, Zhongwei Lin ${ }^{\mathrm{c}}$, Fang Fang ${ }^{\mathrm{c}}$, Qun Chen ${ }^{\mathrm{d}}$

$4{ }^{a}$ Center for Electric Power and Energy (CEE), Department of Electrical Engineering, Technical University of 5 Denmark (DTU), 2800 Kgs. Lyngby, Denmark

$6 \quad{ }^{\mathrm{b}}$ State Key Laboratory of Advanced Electromagnetic Engineering and Technology, Huazhong University of

7 Science and Technology, Wuhan 430074, China

$8{ }^{c}$ School of Control and Computer Engineering, North China Electric Power University, Beijing 102206, China

9 d Key Laboratory for Thermal Science and Power Engineering of Ministry of Education, Department of 10 Engineering Mechanics, Tsinghua University, Beijing 100084, China

11

Email address: menzh@dtu.dk, qw@elektro.dtu.dk, jinyu.wen@hust.edu.cn, 1zw@ncepu.edu.cn, ffang@ncepu.edu.cn, qunchen@tsinghua.edu.cn;

*Corresponding author: Qiuwei Wu, E-mail address: qw@elektro.dtu.dk 


\begin{abstract}
The optimal operation of the integrated electricity and heat systems (IEHS) can bring environmental benefits, reduce the operational cost, and achieve high penetration levels of renewables. This review aims to provide a comprehensive overview of the IEHS modeling and solution methods for the optimal operation. With respect to the optimal operation, the IEHS modeling is reviewed from five aspects, including the representation of dynamic characteristics, operational flexibility improvement, operation under uncertainty, joint dispatch of the electrical power system (EPS) and district heating system (DHS), and joint market clearing of the EPS and DHS. The solution methods for the optimal operation of the IEHS are divided in four groups according to the properties of optimization problems, i.e., methods for integrated energy flow calculation, methods for decentralized optimization, methods using relaxation and convexification techniques, and methods based on intelligent algorithms. The main characteristics, advantages, and limitations of the modeling and solution methods are detailed and compared. In the end, the current gaps and future research are summarized regarding the modeling and solution methods for the optimal operation of the IEHS.
\end{abstract}

\title{
Highlights
}

- The IEHS modeling considers dynamic characteristics, flexibility improvement, uncertainty, and joint dispatch and clearing.

- The IEHS solution methods in different situations

- Current gaps and future research of modeling and solution methods

Keywords: Dynamic characteristic, flexibility improvement, integrated electricity and heat system, joint operation scheme, optimization under uncertainty, solution methods.

\section{Word Count $(12,419)$}

\begin{tabular}{|c|c|c|c|}
\hline IEHS & $\begin{array}{l}\text { Integrated electricity and heating } \\
\text { system }\end{array}$ & COP & Coefficient of performance \\
\hline DHS & District heating system & OCD & $\begin{array}{l}\text { Optimality } \\
\text { decomposition }\end{array}$ \\
\hline EPS & Electrical power system & SOCP & Second-order cone program \\
\hline $\mathrm{CHP}$ & Combined heat and power & MAP & Master problem \\
\hline HPs & Heat pumps & SUP & Sub-problem \\
\hline EBs & Electric boilers & $\mathrm{SP}$ & Stochastic programming \\
\hline STs & Heat storage tanks & $\mathrm{RO}$ & Robust optimization \\
\hline $\mathrm{AC}$ & Alternating current & $\mathrm{DC}$ & Direct current \\
\hline LP & Linear programming & $\mathrm{CCP}$ & Chance constraint programming \\
\hline MILP & Mixed-integer linear programming & ADMM & $\begin{array}{l}\text { Alternating direction method of } \\
\text { multipliers }\end{array}$ \\
\hline
\end{tabular}




\section{DRL Deep reinforcement learning RES Renewable energy sources \\ ML Machine learning}

58

59

60

61

62

63

64

65

66

67

68

69

70

71

72

73

74

75

76

77

78

79

80

81

82

83

84

85

86

87

88

89

90

91

92

93

Contents

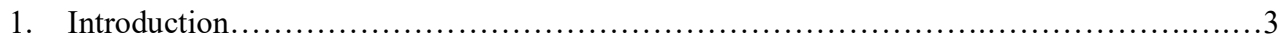

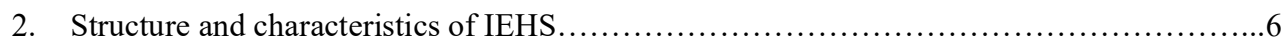

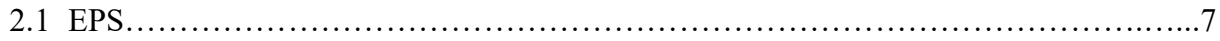

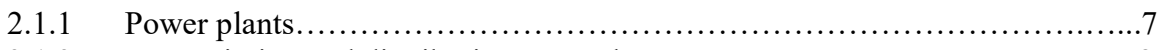

2.1.2 Transmission and distribution networks....................................

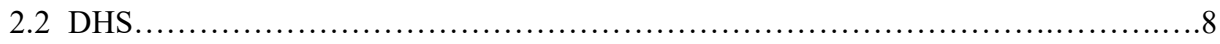

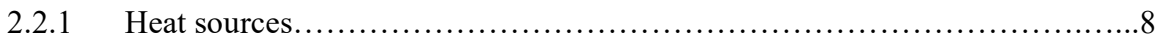

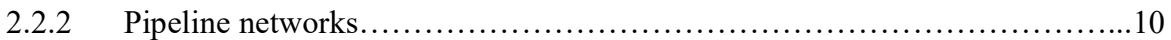

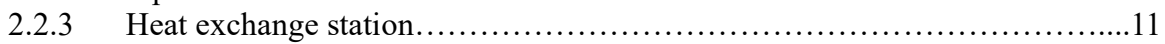

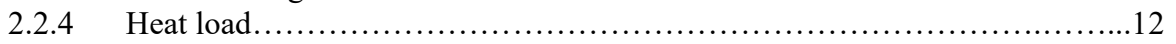

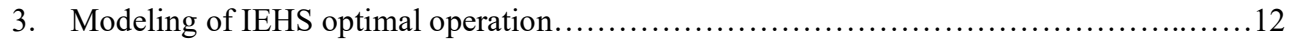

3.1 General formulation of optimal operation of IEHS ............................... 12

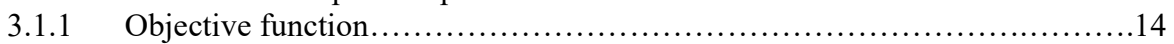

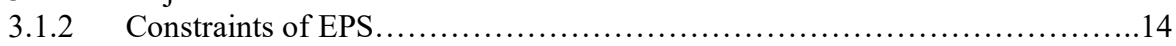

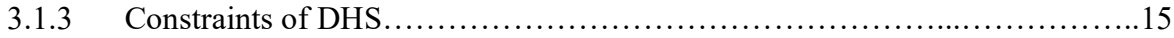

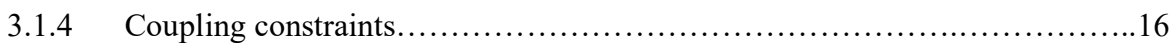

3.2 IEHS modeling ........................................................... 17

3.2.1 Modeling for operation considering dynamic characteristics of DHS .........18

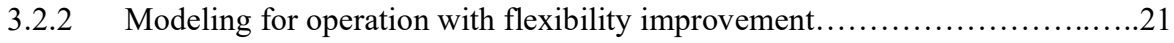

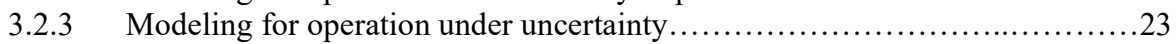

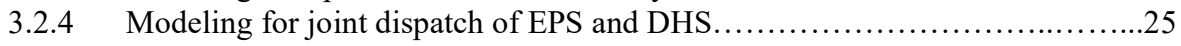

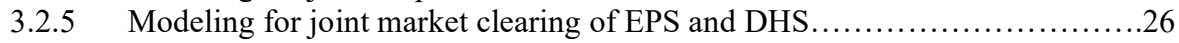

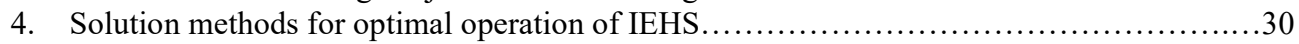

4.1 Solution methods for integrated energy flow calculation.....................................

4.2 Solution methods for decentralized optimization................................. 32

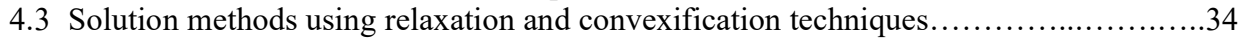

4.4 Solution methods based on intelligent algorithms......................................

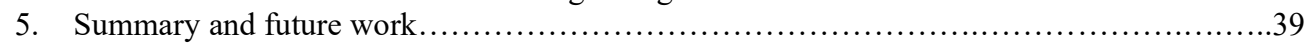

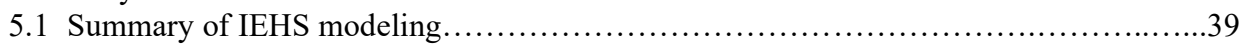

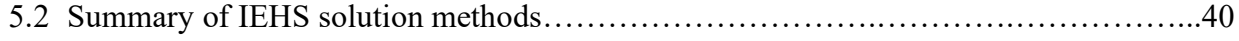

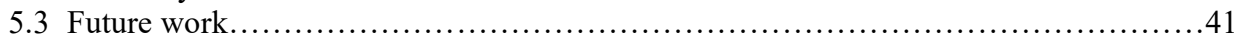

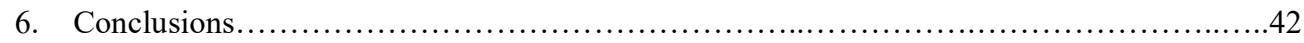

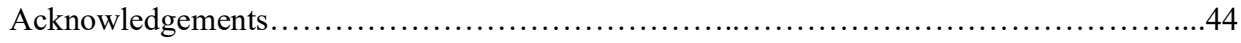

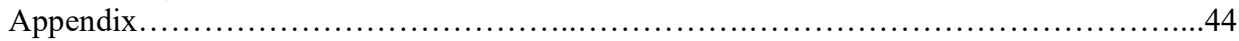

References................................................................ 45

\section{Introduction}

The future energy systems are characterized by a high proportion of renewable energy. In Denmark, the target is to achieve a fully renewable-based energy system by $2050[1,2]$. Renewable energy sources (RES) such as wind and solar power is uncertain and variable [3], which greatly challenges the secure operation of the electrical power system (EPS). Therefore, sufficient flexibility is needed to guarantee the power balance in the EPS $[4,5]$. 
Submitted to Elsevier Science

Among all choices to improve flexibility, the integration with the district heating system (DHS) [6] is a promising way due to its large thermal inertia [7]. To utilize the flexibility of the DHS, the optimal operation of the integrated electricity and heat system (IEHS) has become a research focus. According to recent studies, the optimal operation of the IEHS can bring environmental benefits, reduce the operational cost and improve the utilization of wind power due to its large flexibility $[8,9]$.

The IEHS modeling and solution methods are essential for the optimal operation. The modeling determines whether the operational characteristics and dispatch mechanism of the IEHS can be well reflected. The solution methods affect the computational efficiency and accuracy of the decision-making.

There are several challenges regarding the IEHS modeling for optimal operation. Firstly, it is hard to coordinate the EPS and DHS due to the different dynamic characteristics. As can be seen from Table 1, the dynamic characteristic of the DHS [10] is much slower than that of the EPS. This means the DHS is still in a dynamic process when the EPS reaches a steady state [11]. Therefore, the time delays and thermal dynamic characteristics in the pipelines of the DHS are non-negligible [12]. This also results in the difficulty of choosing a reasonable time resolution in the joint dispatch of two subsystems [13]. Secondly, the diverse flexible devices used for improving the operational flexibility of the IEHS differ greatly in operational characteristics, leading more complicated interactions among devices and subsystems. The coupling devices, such as the combined heat and power (CHP) units, heat pumps (HPs), electric boilers (EBs), interact with the EPS and DHS simultaneously [14]. The heat storage tanks (STs) influence the DHS, which indirectly affect the operation of the EPS [15]. Thirdly, more uncertainty sources and larger uncertainty are introduced into the IEHS, increasing the complexity of the dispatching. The uncertainties from the RES [16, 17], electricity prices [18], and heat demand [19] are all non-negligible in the IEHS. Besides, with the transition to a fully renewable-based energy system, the operation under uncertainty is more challenging both in the uncertainty modeling and balancing measures [20]. Fourthly, the joint dispatch and joint market clearing of the EPS and DHS are not well designed, which hinders the full utilization of the flexibility in the system. For example, the EPS and DHS belong to different operators in Denmark, and the heat dispatch is earlier than the power dispatch [21], which is not global optimal for the interactions with the electricity market [22]. 
Submitted to Elsevier Science

Table 1 Comparisons of dynamic parameters for the EPS and DHS

\begin{tabular}{ccccc}
\hline $\begin{array}{c}\text { Energy } \\
\text { system }\end{array}$ & Carrier of energy flow & Speed & Inertia & Response time \\
\hline EPS & Electricity & $\begin{array}{c}\text { Near speed of } \\
\text { light, } 3 \times 10^{8} \mathrm{~m} / \mathrm{s} \\
\text { Slow, no more } \\
\text { than } 1.2 \mathrm{~m} / \mathrm{s}\end{array}$ & Small & Fast, second level \\
LHS & Heating energy flow & $\begin{array}{c}\text { Slow, hour level to } \\
\text { day level }\end{array}$ \\
\hline
\end{tabular}

The challenges of the IEHS solution methods for optimal operation stem from the following reasons. Firstly, the electrical, thermal, and hydraulic processes are tightly coupled in the IEHS, leading to a large computational burden for the integrated energy flow calculation. Both the integrated and decomposed electrical-thermal-hydraulic solution methods [23, 24] have their limitations. Secondly, the EPS and DHS may belong to different system operators in the joint dispatch or market clearing, where the decentralized optimization method [25] is necessary to achieve the equilibrium among multiple operators. Currently, the decentralized optimization methods differ greatly in different optimization, both for the decomposition of subsystems and the information exchange [26, 27]. Thirdly, the nonlinear terms or nonconvex feasible region caused by device characteristics, network constraints, and optimization under uncertainty make the original problem computationally intractable. For example, the nonconvex operational region of CHP units [28], pressure drop constraints in the DHS [29], the coupling constraint of the mass flow rate and temperature [30], and the max-min operator in uncertainty optimization [31] all bring challenges to the optimization. To make the model tractable, the relaxation and convexification techniques are necessary to cope with these issues. Fourthly, part of the nonlinear optimization is still intractable by only relying on the relaxation and convexification techniques and off-the-shelf commercial solvers. The intelligent algorithms based on group optimization [32] or machine learning (ML) methods [33] can be alternative methods in such situations.

According to the challenges in the IEHS modeling and solution methods, the IEHS modeling is reviewed from five aspects of the optimal operation, including the dynamic characteristics of the DHS, operational flexibility improvement, operation under uncertainty, joint dispatch of the EPS and DHS, and joint market clearing of the EPS and DHS. The solution methods are divided in four groups, including methods for integrated energy flow calculation, methods for 
$6 \quad$ Submitted to Elsevier Science

159

160

decentralized optimization, methods using relaxation and convexification techniques, and methods based on intelligent algorithms.

In order to help researchers build more reasonable models and develop more efficient solution methods, this work reviews the IEHS modeling by focusing on how to coordinate subsystems with different dynamic characteristics, how to model the operational characteristics of flexible devices and their interactions with other parts of the IEHS, how to represent and balance the uncertainties, how to design the joint dispatch and market clearing mechanisms of subsystems. In the meanwhile, the review of the solution methods concentrates on the tradeoff between the tractability, accurate system characteristics, computational burden, computational efficiency, convergence rate, and optimality. The main characteristics, advantages, and limitations of each modeling and solution method are analyzed and discussed.

The rest of the review is organized as follows. Section 2 introduces the structure and characteristics of the IEHS. Section 3 presents the general formulation of the IEHS and the modeling from the five aspects for the optimal operation. Section 4 details four types of solution methods for the optimal operation of the IEHS. A summary and outlook on future research are given in Section 5, followed by conclusions in Section 6.

\section{Structure and characteristics of IEHS}

To achieve the optimal operation of the IEHS, the system operator needs to consider the feasible operational region of the whole system and each component. Therefore, having full knowledge of the structure and characteristics of the IEHS is the base of the modeling and solution methods. Fig. 1 illustrates the general structure of the IEHS, which is composed of the EPS and DHS. The two subsystems are introduced in subsections 2.1 and 2.2, respectively. 


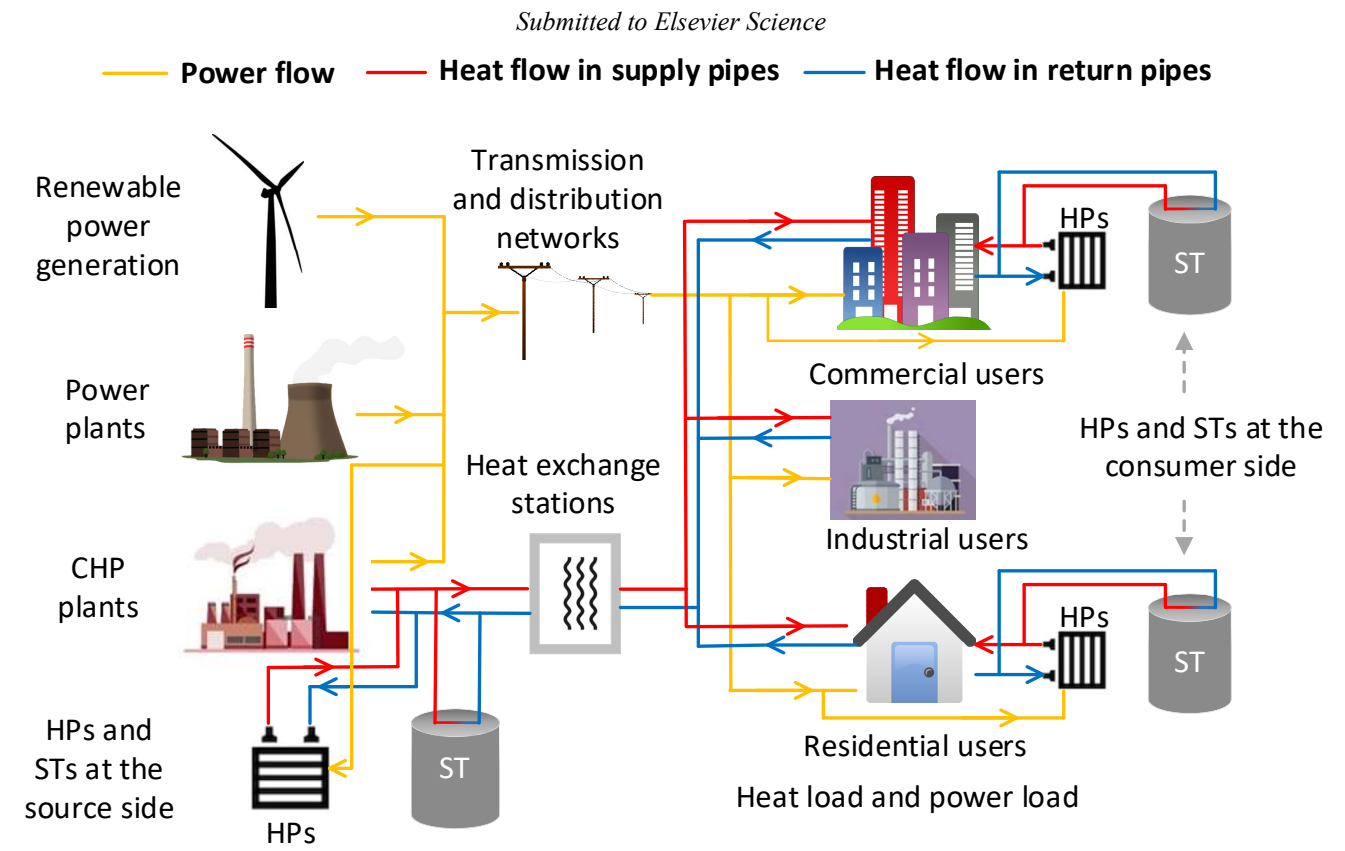

Fig. 1. Structure of the IEHS.

\subsection{EPS}

As shown in Fig. 1, the EPS is a set of all facilities and equipment for the generation, transmission and distribution of electricity.

\subsubsection{Power plants}

The power plants on the generation side are responsible for producing the electrical energy, which can be from thermal, hydraulic, nuclear, wind, solar power generation, etc.

The conventional power units, such as thermal power units, need to consider the maximum and minimum output limits [34], ramping rate [35, 36], reserve capacity [37], and minimum online and offline time [38].

Different from the controllable conventional generators, the wind and solar power generation is non-dispatchable due to the uncertainty of wind and solar radiation [39]. It is necessary to use the flexibility to handle these uncertainties [40]. 


\subsubsection{Transmission and distribution networks}

The electrical energy from power plants is delivered to the users through the transmission and distribution networks [41]. In the EPS, the decision-making for the optimal operation of the transmission and distribution networks is usually separated with different system operators [42]. The direct current (DC) power flow method is widely utilized to model the transmission and distribution network constraints, which limits the power flow on a line not exceeding its capacity $[43,44]$. Other research utilizes the alternating current (AC) method to model the power flow $[45,46]$, which is more accurate than the DC method while having more complex constraints.

\section{2. $D H S$}

A detailed structure of the DHS is illustrated in Fig. 2, which includes the heat source, pipeline network, heat exchange station, and heat load [12]. The following subsections describe these four parts.

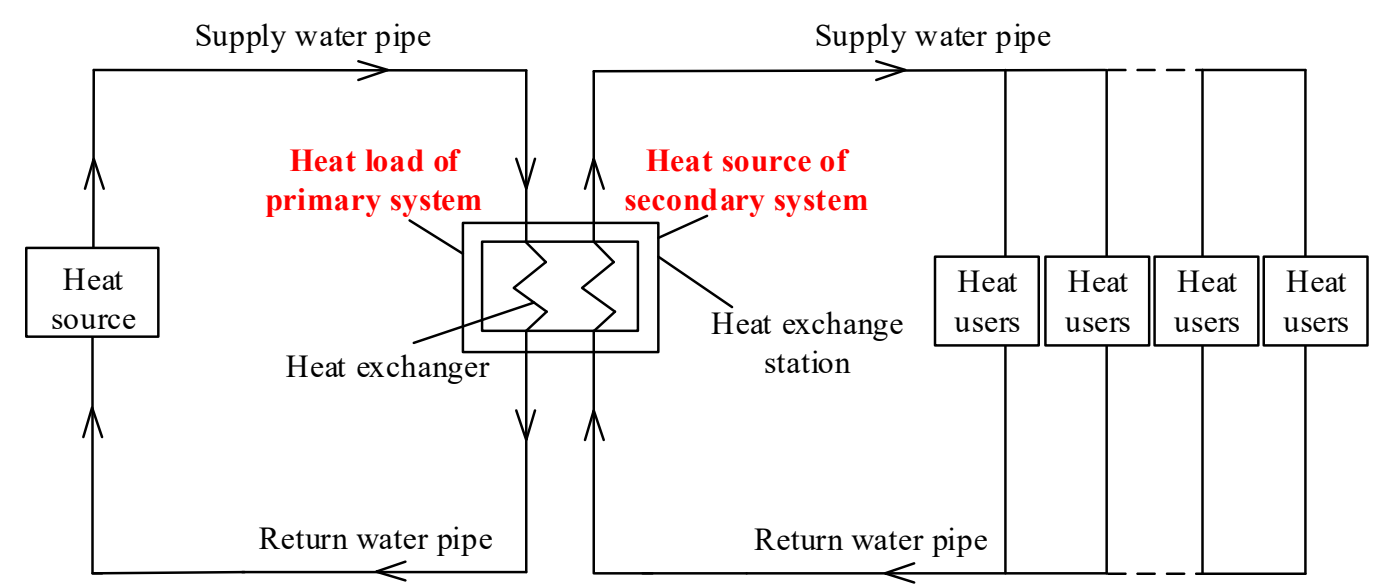

Primary system

Secondary system

Fig. 2. Structure of the DHS

\subsubsection{Heat sources}

CHP units are the main heat sources in the DHS. According to the difference of exhaust steam pressure, CHP units can be classified into the back-pressure unit and condensing unit.

Table 2 summarizes the main differences between the two types of CHP units for the structure, energy efficiency, power and heat production relationship, and unit capacity. Those differences lead to different models of them. 
The back-pressure CHP units use low-pressure steam for heat supply [47] while condensing CHP units have one or more openings in its casing to extract steam, delivering both low pressure and medium pressure steam for heat supply [48].

As shown in Table 2, the back-pressure CHP units have higher energy efficiency than the condensing CHP units [49]. This is because all the exhaust steam of back-pressure turbines is utilized for heat supply. However, the back-pressure CHP units provide little flexibility to meet electrical loads due to the fixed power-to-heat ratio [50]. In comparison, the condensing CHP units are more flexible in adjusting power supply with a variable power-to-heat ratio [51]. As the back pressure increases, the generation capacity will be reduced. Therefore, the capacity of back pressure CHP units is usually smaller than the one of condensing CHP units.

Table 2 Comparison between two types of CHP units

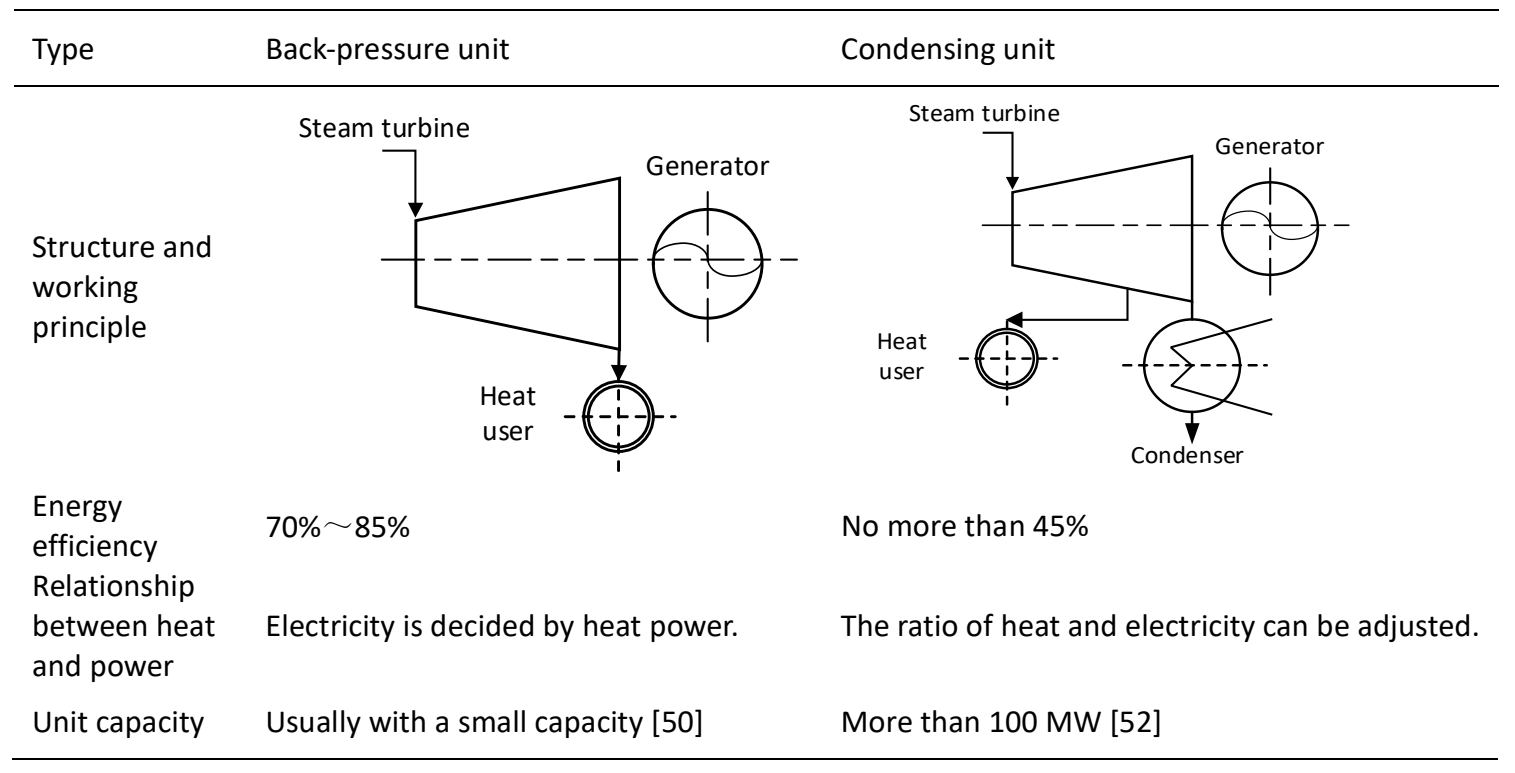

Besides, CHP units can be connected to different grid levels. The centralized CHP units are connected to the DHS at the transmission level; the decentralized CHP units are connected to the DHS at the distribution level; the micro-CHP units are utilized at the building level [4].

Apart from the CHP units, the HPs, EBs, and STs could also provide heat for the DHS as auxiliary heat sources. The EBs consumes electrical energy to generate heat with a relative low energy conversion efficiency, usually around 0.9 [53]. Ref. [54] introduces the operation 

principle of an HP system. Approximately $75 \%$ of the energy sources of an HP come from the air, ground, or water, and about $25 \%$ of the total energy is used to transform the energy from nature to useful heat. Therefore, the HPs usually have a higher energy efficiency than EBs. The STs release the stored energy to provide heat.

\subsubsection{Network of pipelines}

Similar to the EPS, the DHS can also be divided into a transmission system (primary pipe network) and a distribution system (secondary pipe network), as shown in Fig. 2.

The primary pipe network refers to pipelines that transfer heat from the heating plant to the heat station. The secondary pipe network refers to pipelines that transfer heat from the heat station to the heat user. The water in primary and secondary pipelines is physically isolated by the heat exchange station [26], which returns water back to the heat source directly in the primary network. With the physical isolation, the two networks can have different mass flow rate and pressure.

The temperature and pressure of the DHS usually vary from country to country. In most primary networks, the maximum supply water temperature is not more than $140^{\circ} \mathrm{C}$. Most of the primary heat networks operate at below $140{ }^{\circ} \mathrm{C}$ and some of them operate at even below $80{ }^{\circ} \mathrm{C}$ [55]. The primary return water temperature ranges from $35{ }^{\circ} \mathrm{C}$ to $55^{\circ} \mathrm{C}$ [56]. In Denmark, the supply and return temperature of the secondary network is around $70{ }^{\circ} \mathrm{C}$ and $40{ }^{\circ} \mathrm{C}$, respectively.

The pressure of the primary network can reach 25 bar. In most cases, the pressure is below 16 bar. In the secondary networks, the pressure of the connected buildings is from 6 to 10 bar [55].

Fig. 3 is a general structure of the primary pipelines to illustrate the operational characteristics of the DHS, which are outlined as follows:

- The pipeline network has two processes, i.e., the hydraulic process and thermal process. The hydraulic process relates pressures and mass flow rates, and the thermal process is about temperature changes and heat exchange. These two processes are tightly coupled.

- In any loop composed of water pump and pipes, the pressure drop is zero. For any node, the outflow equals inflow.

- The mass flow rate and pressure drop in supply and return pipes are symmetrical, which means $M_{i, j}^{\mathrm{S}}=M_{i, j}^{\mathrm{R}}$ and $P_{i}^{\mathrm{S}}-P_{j}^{\mathrm{S}}=P_{j}^{\mathrm{R}}-P_{i}^{\mathrm{R}}$, where $i$ and $j$ are neighboring nodes, $M^{\mathrm{S} / \mathrm{R}}$ is 

the mass flow rate of supply/ return pipes, and $P^{\mathrm{S} / \mathrm{R}}$ is the node pressure of supply/ return pipes.

- For a pipeline, its inlet temperature is higher than outlet temperature due to the heat loss during the transmission, which means $T I_{i, j}^{\mathrm{S} / \mathrm{R}}>T O_{i, j}^{\mathrm{S} / \mathrm{R}}$.

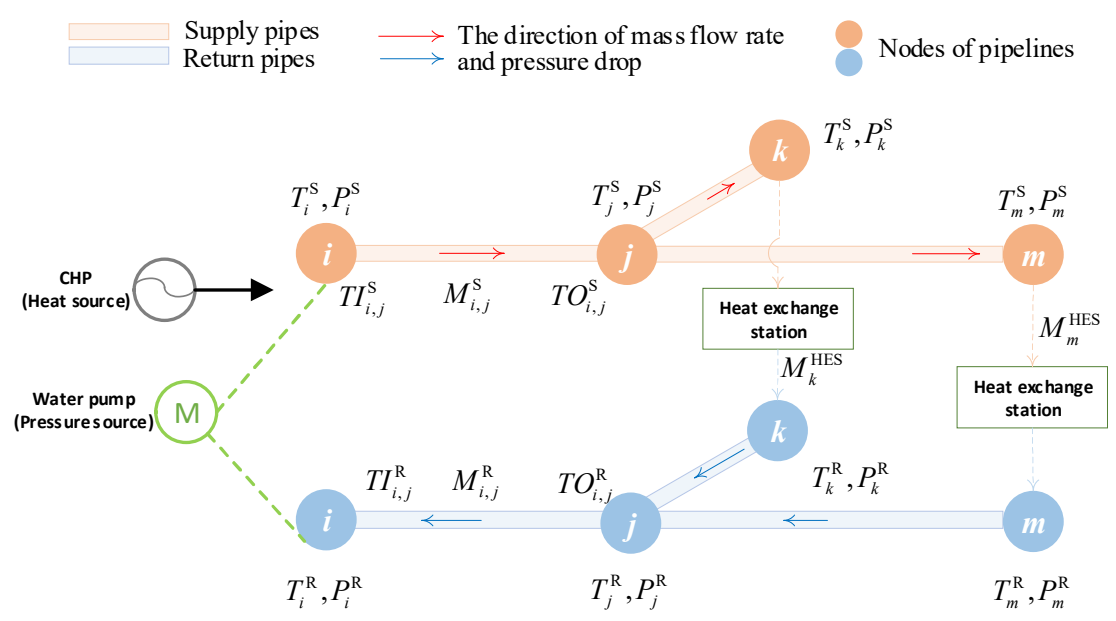

Fig. 3. General structure of the primary pipelines.

\subsubsection{Heat exchange station}

The heat exchange station is mainly used to reduce the water temperature in the primary pipelines so as to reduce heat loss during heat transfer will increase.

Located at the end of the primary network and being responsible for the heat transfer, the heat exchange station is a heat load of the primary network [12], as illustrated in Fig. 2. The heat exchange station allocates the obtained heat to different users in the secondary network and is a heat source of the secondary network.

\subsubsection{Heat load}

The heat load in the central heating network can be the buildings in a neighborhood, town center or whole city. It is determined by the difference between indoor and outdoor temperatures [52]. Ref. [57] utilizes a discrete differential equation to model the heat load with the difference between the indoor and outdoor temperatures incorporated. Ref. [58] utilizes population 
distribution data and the surface temperature data from the Modern Era Retrospective Analysis to determine the heat load within each city.

The heat load mainly has two features.

- Firstly, the heat load is usually considered as changing linearly with the outdoor temperature and the changes for all local heat loads in a network are simultaneous.

- Secondly, the proportion of any local heat load in the total heat load is constant when neglecting the small disturbances on the user side, such as the window opening behavior. The proportion does not change with the outdoor temperature.

\section{Modeling of the IEHS optimal operation}

\subsection{General formulation of optimal operation of IEHS}

A model for the optimal operation of the IEHS is composed of the objective function, constraints of the EPS and DHS networks, and coupling constraints of the EPS and DHS, as shown in Fig. 4.

\section{Modeling of optimal operation of IEHS}

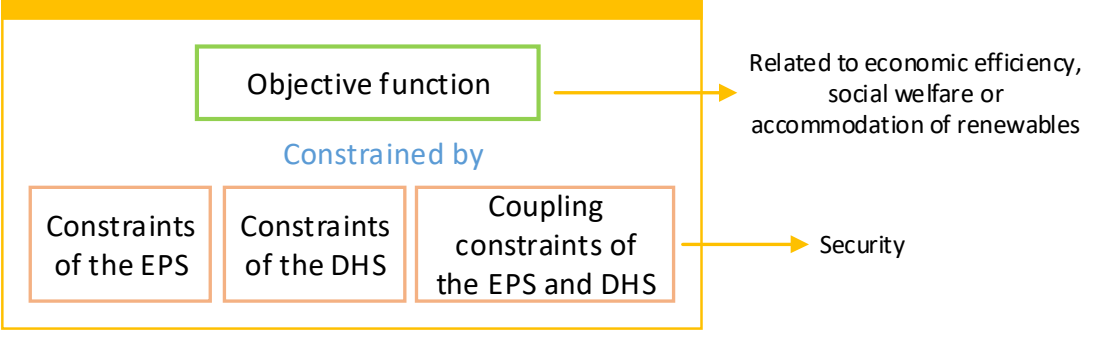

Fig. 4. Composition of a model for the optimal operation of the IEHS

The objective function usually reflects the system operational cost, while the constraints decide how accurately and efficiently the characteristics of the IEHS have been formulated. The objectives are usually about the optimal operational cost, maximum social welfare, maximum utilization of RES. The constraints represent the feasible region of each device and the whole system.

Take the modeling of the IEHS in the transmission network as an example. The general formulation is summarized in Table 3 . 


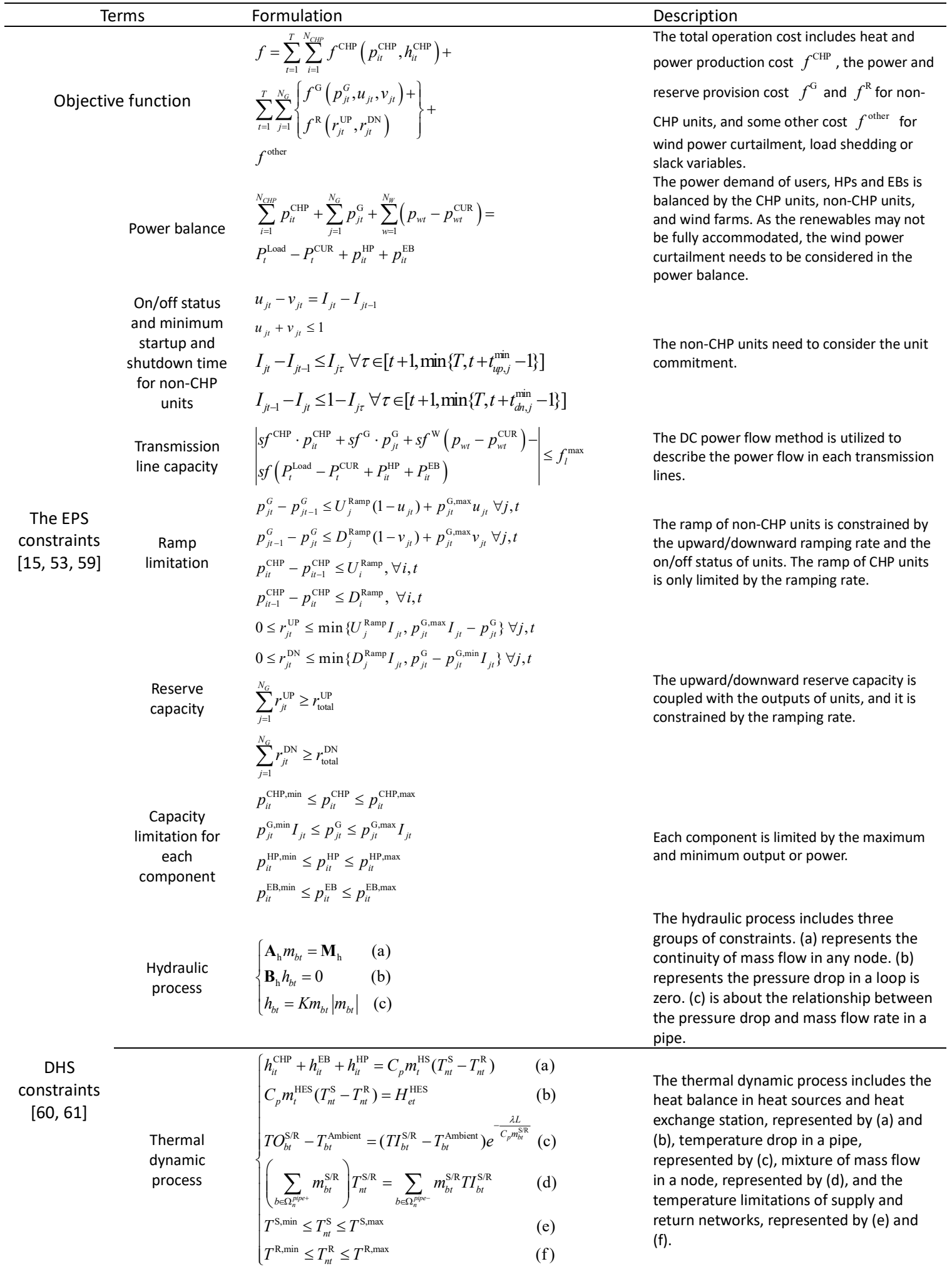




$$
\begin{array}{lll}
\begin{array}{c}
\text { Coupling } \\
\text { constraints } \\
\text { of the EPS } \\
\text { and DHS }
\end{array} & \text { CHP } & h_{i t}^{\mathrm{CHP}}=g\left(p_{i t}^{\mathrm{CHP}}\right) \\
{[62]} & \text { HPs } & h_{i t}^{\mathrm{HP}}=\eta^{\mathrm{HP}} p_{i t}^{\mathrm{HP}} \\
& \text { EBs } & h_{i t}^{\mathrm{EB}}=\eta^{\mathrm{EB}} p_{i t}^{\mathrm{EB}}
\end{array}
$$

The relationship between heat and power production of CHP units, which differs when the unit type is different. The constraints for CHP will be detailed in the subsection 3.1.4.

The relationship between heat production and power consumption for HPs.

The relationship between heat production and power consumption for EBs.
310

It should be noted that the formulation in Table 3 is just a fundamental form. It can be revised or simplified when the operational environment changes, such as the joint operation modes of subsystems, owners of flexible devices, voltage level of the EPS and so on. These formulations can be changed when the modeling focuses on different aspects. The further explanation for the formulation is described in the following subsections.

\subsubsection{Objective function}

The cost items in the objective function in Table 3 includes the start-up cost, power and heat production cost, penalty cost of wind power curtailment, reserve cost, penalty cost of slack variables, and so on.

For the joint operation of the EPS and DHS, when the operation mode of multiple subsystems is different, the objective function in Table 3 changes. For example, when the joint operation mode of subsystems is based on the centralized operation, the cost relates to both power and heat production, while when the joint operation mode is based on the decentralized operation, the cost is divided into two parts according to the entities where the cost belonged to.

When the electricity market is accounted for, the objective function is related to the maximum welfare instead of the fuel cost. In the market without unit commitment, the startup cost of units will not be included. Therefore, the objective function should be set according to the operational condition of the system.

\subsubsection{Constraints of EPS}

Due to the diverse market setup and different joint operation modes of subsystem, the security constraints that need to be considered in the EPS are different. For example, in the power grids of China [63] and the PJM market [64] in the USA, unit commitment is considered. Therefore, the on/off status and minimum startup and shutdown time constraints will be necessary. 
However, in some other places, such as the Nordic electricity market, only the constraints of economic dispatch are needed.

The constraints that all systems must consider are the power balance constraints and transmission line constraints. The power flow constraints in Table 3 is based on the linear DC power flow method, which is widely utilized in the transmission network due to its high accuracy of approximation. At the distribution level, the nonlinear AC power flow is also a common method to model the energy flow [65].

\subsubsection{Constraints of DHS}

The heat network model has two processes, i.e., the hydraulic process and thermal process. The hydraulic process is about the fluid condition, and the thermal process is about temperature changes and heat exchange [29]. These two processes are tightly coupled, leading to a highly nonlinear model of the DHS.

For the modeling of the hydraulic process in Table $3, \mathbf{A}_{\mathrm{h}}$ is the node-branch incident matrix, $m_{b t}$ is the mass flow rate of pipeline $b$ in period $t, \mathbf{M}_{\mathrm{h}}$ is the outflow of each node, $\mathbf{B}_{\mathrm{h}}$ is the loop-branch incident matrix, $h_{b t}$ is the pressure drop in pipeline $b$ in period $t$, and $K$ is the resistance coefficient.

For the modeling of the thermal process in Table $3, T_{b t}^{\text {Ambient }}$ is the time-varying ambient temperature, $T I_{b t}^{\mathrm{S} / \mathrm{R}}$ and $T O_{b t}^{\mathrm{S} / \mathrm{R}}$ are temperatures at the beginning and end of a pipeline, $\lambda$ is the insulation coefficient of the pipes, $L$ is the length of the pipes, and $T_{n t}^{\mathrm{S} / \mathrm{R}}$ is the temperature of node $n$ after mixing.

It should be noted that the hydraulic and thermal constraints in Table 3 are mainly for the primary heat network. For the secondary heat network of the DHS, little work has considered its modeling due to the widely distributed heat consumers, extremely complicated topology, and rare measuring points in the secondary pipelines.

Except for the basic hydraulic and thermal constraints in Table 3, recent studies have devoted to formulating more accurate network constraints. Ref. [29] considers the flow direction in the pipelines by introducing binary variables to represent flow direction. Ref. [66] accounts for the 
time delay of temperature changes in mass flows by formulating temporal coupling constraints in the DHN.

\subsubsection{Coupling constraints}

As can be seen from the coupling constraints listed in Table 3, the EPS and DHS are mainly coupled by CHP units and other flexible devices such as HPs and EBs. Table 3 gives the coupling constraints of HPs and EBs. This section only details the constraints of CHP units.

As stated in Section 2.2.1, CHP units are mainly classified into the back-pressure unit and condensing unit. Table 4 shows their feasible region of heat and power production. The details can be found in [67] for the feasible region of CHP units.

Types
Condensing type with
convex feasible region
Condensing type with
nonconvex feasible
region


373 For back-pressure units, the electricity production changes linearly with heat production, as 374 shown in Table 4.

375 For condensing units, its feasible region is surrounded by several operational curves. When 376 the feasible region is convex, the linear combination of the convex hull's extreme points can be 377 utilized to describe its feasible region $[15,68]$.

378 In contrast, if the feasible region is nonconvex, it will be firstly divided into two convex sub379 regions 1 and 2 . Then each sub-region is modeled with the traditional method and they are 380 combined by introducing binary variables $I_{1}^{\mathrm{CHP}}$ and $I_{2}^{\mathrm{CHP}}$. Refs. [28] and [69] give detailed 381 models for the nonconvex feasible region of the CHP unit.

382 In summary, the modeling of CHP units depends on the actual type in the system. The 383 modeling of back-pressure and condensing CHP units with the convex feasible region is in linear 384 forms, while the condensing units with the nonconvex feasible region is in a mixed-integer form.

385 3.2. IEHS modeling

386 Fig. 5 summarizes the IEHS modeling for optimal operation from five aspects, i.e., the 387 operation considering dynamic characteristics of the DHS, operation with flexibility 388 improvement, operation under uncertainty, joint dispatch of the EPS and DHS, and joint market 389 clearing of the EPS and DHS. 


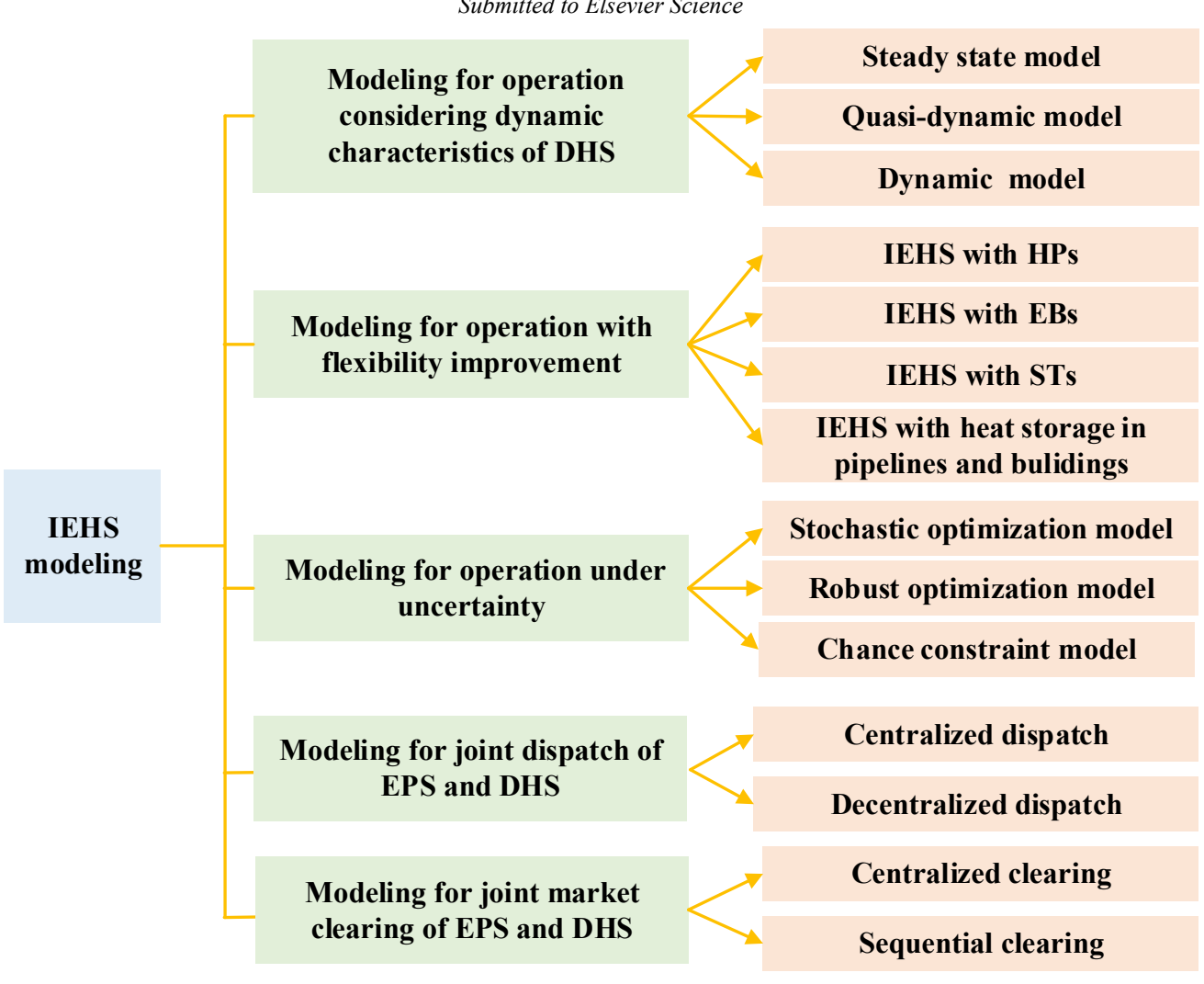

Fig. 5. IEHS modeling in optimal operation for different situations

\subsubsection{Modeling for operation considering dynamic characteristics of DHS}

As mentioned in the introduction, the speed of electrical energy is near the light, which means the power flow is instantaneously balanced in the EPS. The rate of heat transfer in the DHS is much slower than the speed of electrical energy. This means that the former is still in a dynamic process when the latter reaches a steady state. Therefore, it is not necessary to consider the dynamic characteristics of the EPS during the power balance while it is non-negligible to consider the dynamic characteristics of the DHS in the heat transfer. Neglecting the dynamic characteristics of the DHS may result in an infeasible or suboptimal solution.

According to whether the dynamic characteristics of the hydraulic process and thermal process in the DHS is considered, the IEHS modeling can be classified into three categories. Fig. 6 compares the main difference between them. 
The steady-state model usually neglects the time delays and thermal dynamics in the pipelines [70], as well as the variable mass flow rate. Refs. [66] and [71] only consider the balance between the heat demand and heat production when formulating the steady-state model. Although the model is easy to be established and computationally tractable, it may fail to give a realistic result after neglecting the dynamic characteristics.

In contrast, the quasi-dynamic model considers the time delays and thermal dynamics in the pipelines. The temperature of the pipeline changes with time and location. Essentially, the pipeline temperature is a binary partial differential equation with respect to time and location. However, the IEHS modeling based on the partial differential equation is computationally intractable. To handle this challenge, Ref. [11] utilizes Taylor expansion and finite differential method to discretize the partial differential equation of pipelines into nonlinear algebraic equations, by choosing a time step $\Delta t$ and distance step $\Delta x$. Fig. 7 shows the basic idea of the finite difference method.

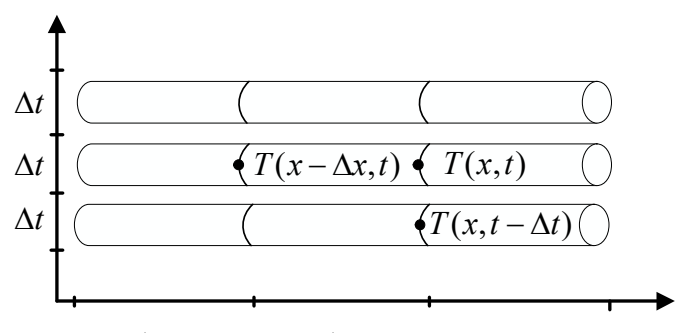

$\Delta x$

$\Delta x$

$\Delta x$

Fig. 7. The basic idea of finite differential method

Most research $[7,29,60]$ neglects the distance step $\Delta x$ when building the quasi-steady model because the mass flow rate is usually assumed to be a constant. However, it is still a challenge to choose a reasonable time step $\Delta t$ for the DHS. On the one hand, the time step represents the 
time resolution of scheduling. When the time step of the DHS differs from that of the EPS, the synergy of different time resolutions of scheduling for two subsystems will be a challenge. On the other hand, the time step influences the approximation accuracy and the computation burden simultaneously. A large time step may cause the heat imbalance in the source side and consumer side due to the low approximation accuracy for the time delay and thermal dynamic characteristic in the pipelines. In contrast, a fine time step may bring a high computation burden.

At present, most research sets the time resolution of the DHS scheduling as the same with the EPS, little work has considered how to identify the optimal time resolution for the DHS. Ref. [13] investigates the influence of the time resolution on computational accuracy and efficiency. Other research about the quasi-dynamic model focuses on describing the time delay of transmission and the thermal dynamic characteristic. Ref. [26] models the temperature mixing and heat loss in the same equation. Besides, it builds the relationship between the temperature of a node and its child nodes' temperatures of past periods to reflect the time delay characteristic. Refs. [19] and [72] apply the node method to model the quasi-dynamics of temperature.

In the DHS, there are two regulation modes, i.e., quality regulation and quantity regulation. The former has a constant mass flow rate and variable supply temperature, while the latter has a variable mass flow rate and constant supply temperature. A lot of research on the quasidynamic model is based on the quality regulation [73], and other research considers the quantity regulation [74].

The dynamic model considers the variable mass flow rate on the basis of the quasi-dynamic model. In the hydraulic process, the mass flow rate is adjusted by the opening degree of the regulating valve and the circulating pump, the modeling of which is usually complicated. Refs. $[75,76]$ propose a dynamic model of the DHS, which considers the variable mass flow rate. However, this model does not combine the EPS and DHS. When the EPS and DHS are integrated, the complexity of calculation will not be avoided due to the highly nonlinear characteristic of the hydraulic process. Therefore, it is still a great challenge to apply the dynamic model due to the requirement of computational efficiency.

In summary, most of the current research is based on the quasi-dynamic models due to its tradeoff between the accuracy and computational efficiency. 


\subsubsection{Modeling for operation with flexibility improvement}

Improving the operational flexibility is an important aspect of the optimal operation of the IEHS, which can help reduce energy cost and increase accommodation of renewables. Different flexible devices, such as the EBs, HPs, STs, and heat pipes, can be used to improve operational flexibility. However, due to different operational characteristics and interactions with other parts of the IEHS, these flexible devices should be modeled differently. For example, the HPs and EBs can provide flexibility by changing their energy consumption behaviors, while the STs provide the flexibility for the DHS by heat load shifting. The pipelines can be seen as a special heat storage device to provide flexibility due to its thermal inertia. These differences can further influence the other parts of the IEHS, such as the operational region of CHP units, and energy balance and transmission.

\section{IEHS modeling with STs.}

The modeling of heat storage devices usually considers three sets of constraints, the heat energy levels, maximum energy capacity, and heat charging/discharging rate.

Ref. [29] utilizes heat accumulators and waste heat from industrial activities to harvest the extra flexibility from the DHS by considering the three sets of constraints. Ref. [15] proposes a more detailed model of STs, considering the mixing of hot water and cold water in the processes of heat storage and heat discharging. Apart from the three sets of constraints, Ref. [28] considers the heat loss and extra heat generation of a heat buffer in a micro-grid with CHP units. Ref. [77] proposes a multi-scale model of STs, which is based on the partial differential equation. However, such a model is highly nonlinear, limiting its application in the modeling of the IEHS.

\section{IEHS modeling with EBs.}

Even without high energy efficiency as the HPs, the EBs are low-cost solutions. In Ref. [72], the heat output of an EB is linearly related to electricity consumption by a constant converting efficiency. Besides, the electricity consumption of the EB has a capacity constraint. Considering the local market policy, Ref. [62] formulates the total operational cost model of EBs with the subsidy and tax cost included. Indices for quantifying flexibility provided by EBs and HPs are proposed in Ref. [78] based on their technical constraints and operating points.

\section{IEHS modeling with HPs.}

The flexibility of HPs is a highly discussed topic both at the consumer side and grid side. 
At the consumer side, domestic HPs have been researched for their flexibility function. Ref. [79] reviews the HP system at the building level and their applications for voltage control, congestion management and provision of spinning and non-spinning reserve. Ref. [80] introduces metrics applicable for different stakeholders to quantify building-to-grid demand response flexibility from the HP aggregation. Ref. [81] has a debate on the energy efficiency and economic potential of flexible HP control methods, which points out installing HP in domestic is hard to achieve cost savings at present. The existing research usually utilizes the first order differential equation of temperature to model the residential HPs [82], in which the HPs adjust the temperature by changing its on/off status. Ref. [83] explores the opportunity to couple CHP and HPs by modeling new energy efficiency equations. With the fast increase of HPs in Denmark, Ref. [84] optimizes the heat usage in a single-family house to achieve the minimum cost of electricity usage according to the forecasted weather and energy prices.

At the grid side, centralized HPs are usually coordinated with the CHP units to provide flexibility for the IEHS. Centralized HPs are usually on a large scale, about $660 \mathrm{MW}$ of heat pumps were operational in Stockholm of Sweden in 2016 [85]. Ref. [86] shows that large-scale HPs are especially promising for efficiently reducing the wind power curtailment.

In Europe, most of the HP systems are connected to a hydronic system and a thermal storage tank, which are used for heating buffer [87]. In Germany, about $90 \%$ of the existing and $80 \%$ of the newly built buildings are equipped with a thermal storage tank when an HP unit is used. Therefore, this fact should also be accounted for when modeling the HPs [88].

The coefficient of performance (COP), which is defined as the ratio of usable heat and the needed electricity. The modeling of COP differs for HPs with different energy sources. For example, the COP of an air-source HP is strongly dependent on the ambient temperature, which is not a constant as the ground-source HP. Ref. [27] utilizes piecewise linear approximation to model the COP of an HP.

\section{IEHS modeling with heat storage by pipelines and buildings.}

Apart from the above devices, the heat storage of pipelines could also provide flexibility. Unlike the EPS, the heat change in the DHS is a slow process. The balancing process between heat production and heat demand will cover several dispatch intervals. Consequently, the heat storage property of the pipeline can be exploited to enhance the flexibility of the DHS. Refs. 
515 [30] and [61] exploit the energy storage capability of a district heating network to tackle the lack 516 of flexibility.

517 However, the time delay in the pipeline network makes the pipeline storage hard to be used as a storage unit. Compared with pipeline storage, the building storage is a more feasible way due to the direct interaction with heat consumers. Some work formulates the building storage model for the optimal operation of the IEHS, by utilizing the equivalent thermal parameter method and allowing the temperature of heat consumers to fluctuate within a predefined range $[7,73]$.

\subsubsection{Modeling for operation under uncertainty}

The uncertainties in the IEHS are from multiple sources, such as renewable energy, heat demand, electricity prices, etc. To guarantee the secure operation of the IEHS, the system operator should account for all the possible uncertainties which may occur in real-time. The stochastic programming (SP) [89] and RO [90] are the two most popular decision-making tools under uncertainty.

Fig. 8 compares the main difference between the RO and SP. Generally, the SP adopts a scenario set to describe the uncertain characteristic of renewable energy. It minimizes the expected total cost under all scenario constraints. The SP has the challenge of balancing computation accuracy and efficiency [59]. In contrast, the RO usually models the uncertainty as an easy-to-obtain box set with budget constraints but suffers from the conservative solution [91].
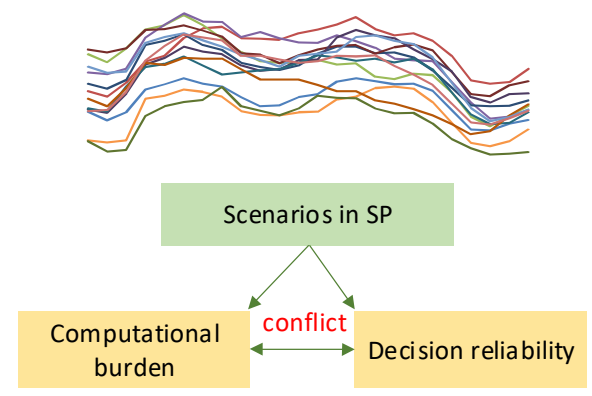

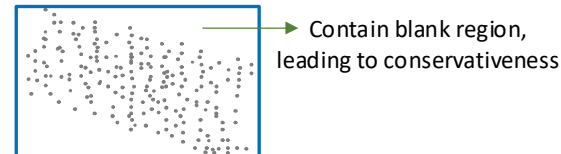

Uncertainty set in a specific shape in RO

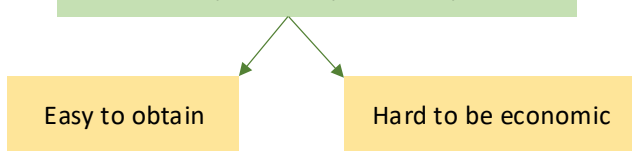

Fig. 8. The main difference between the RO and SP

The modeling based on the SP technique for the optimal operation of the IEHS usually utilizes two-stage or multi-stage framework to bridge the links between day-ahead and real-time 
operation. Ref. [92] formulates a two-stage SP model that bridges the day-ahead and real-time operation in multi-energy systems, in which the uncertainty of wind power is depicted by a set of generated scenarios. Ref. [93] adopts the two-stage SP to build the market clearing model so as to alleviate the operational risk associated with uncertainties of renewable energy in the IEHS. Ref. [39] utilizes a finite set of scenarios to model uncertainties of wind power, electricity demand, and participants' offer. Ref. [94] formulates a day-ahead optimization model based on the two-stage SP, considering uncertainties of outdoor temperature, electricity and hot water consumption, dwelling occupancy, and imbalance prices. Different from the traditional twostage SP, Ref. [95] proposes a three-stage SP model for the short-term operation scheduling of a CHP system, where the uncertainties of day-ahead electricity prices and heat demand are included in the form of scenarios.

A scenario generation method accounting for the correlations of renewables is proposed in Ref. [96], which is validated effective in reducing the operation cost of the EPS. Ref. [14] utilizes the Gaussian mixture model and exponential function method to generate wind power scenarios with spatial and temporal correlation in the decision-making of the stochastic optimal operation of the IEHS. However, only the correlation between wind farms is considered.

In the IEHS modeling based on the RO, the affinely adjustable RO and two-stage adaptive RO are the two mainstream modeling approaches accounting for uncertainties. Ref. [97] formulates the IEHS model based on the affinely adjustable RO, considering the uncertainties of electricity price and heat demand. Usually, the regulation of units is a linear function of the uncertain parameters in the affinely adjustable RO model. Therefore, models based on the affinely adjustable RO are computationally tractable. Refs. [19] and [98] utilize a two-stage adaptive RO model to exploit the influence of uncertainties of the DHS on the operation of the IEHS. Ref. [19] considers the uncertainties pertaining to pipeline parameters and ambient temperature. Ref. [98] considers uncertainties of solar radiation and electricity and heat demand with predefined scenario sets. Besides, the N-1 constraints of the worst-case scenario are also considered to guarantee robust and feasible solutions. The two-stage adaptive RO model has a more complicated structure than the affinely adjustable RO, but it characterizes more flexibility to regulate the day-ahead operational point in the real-time recourse stage.

To reduce the conservativeness of the traditional RO, several measures have been proposed, such as advanced uncertainty sets, the distributionally RO, as well as RO variants. The advanced 
uncertainty sets [99-102] reduce the conservativeness of the RO by accounting for the spatial or temporal correlations of renewables. However, the exploitation of advanced uncertainty sets is mainly used in the EPS. The distributionally RO reduces the conservativeness by minimizing the expected total operation costs under the worst-case distribution instead of the worst-case scenario [103, 104]. Ref. [105] applies this method in the IEHS modeling considering wind power uncertainties. A variant of RO, namely, the information gap decision theory, is proposed in Ref. [106] to evaluate a profitable operation strategy for CHP units in the electricity market. This method reduces the conservativeness by limiting the minimum required or preferred reward value of the objective.

Apart from the popular SP and RO decision-making methods, a few studies utilize the chance constraint programming (CCP) method to cope with the uncertainty in the IEHS. Ref. [107] utilizes the CCP to obtain the optimal energy flow of the IEHS under the uncertainty of electricity, heat loads and solar power. In the modeling for $\mathrm{CCP}$, the security constraints are satisfied with a predefined probability. The nature of the CCP is similar to the SP, which also utilizes the scenarios to represent the uncertainty. Therefore, this method is not extensively reviewed.

\subsubsection{Modeling for joint dispatch of EPS and DHS}

In the operating environment without market setup, the schedule is made by the dispatch center. According to whether the EPS and DHS belong to the same dispatch center, the joint dispatch of the EPS and DHS can be divided into centralized and decentralized dispatch.

In the centralized dispatch, the EPS and DHS belong to the same dispatch center, which has the whole system's information and can dispatch the EPS and DHS simultaneously to fully utilize the flexibility from the DHS. This may be practical for a small microgrid managed by a single control center. However, in a larger area, such as at a city level, there may be multiple DHSs, and the DHS and EPS usually belong to different control centers. For those who do not want to disclose and share their information, it is infeasible to perform the centralized optimization.

To solve this challenge, Ref. [108] develops a new dispatch scheme in the modeling from the perspective of the EPS, which not only considers flexibility utilization from the DHS but also accounts for the private information protection of the DHS. In the proposed method, the operator 
of the DHS sends a modified model of flexible feasible region to the dispatch center of the EPS in a more condensed and privacy-preserved way, but still provides enough information for the EPS dispatch center to complete the integrated dispatch.

Some other researchers are more inclined to establish a decentralized dispatch scheme to use DHS's flexibility, due to the fact that the EPS and DHS still belong to different entities at the current stage. Ref. [76] models two sub-problems, namely, the EPS sub-problem and DHS subproblem, based on the decentralized operation. Each subsystem iteratively exchanges information with others to complete the integrated dispatch. Eventually, each subsystem achieves its optimal operating point and meets the flexibility requirement. The key point in the decentralized dispatch is to design the exchange information in a way to have better privacy protection and less communication burden.

\subsubsection{Modeling for joint market clearing of EPS and DHS}

The IEHS modeling for joint market clearing of the EPS and DHS can be divided into sequential clearing and decentralized clearing according to the clearing order of the two subsystems.

Take Denmark as an example of the sequential clearing. At present, the electricity market and heat market are independent, run by different utilities. The electricity trade is cleared in the Nord Pool, while the heat dispatch is run by the Varmelast.dk [21]. In the Nordic electricity market, the day-ahead market is cleared at noon, during which the market operator runs a market clearing process aimed at maximizing social welfare dispatch to determine the prices for participants [109].

The daily heat plans in the Greater Copenhagen area are generated based on the procedure in Fig. 9 with communication between CHP plants and Varmelast.dk, an independent market operator. Ref. [110] describes this procedure in detail. In Fig. 9, the heat dispatch is determined by Varmelast.dk before 9:00 am. The Varmelast.dk determines the least cost heat dispatch to meet heat demand according to the heat bid curve, which includes the available capacities and marginal cost of each CHP plant. With the received heat order, the heat producers send detailed plans to Varmelast.dk for hour-by-hour heat production of each unit and marginal prices for changing heat production. At last, the Varmelast.dk makes the final heat plan considering the 

ahead electricity market clearing.

632
VARMELAST

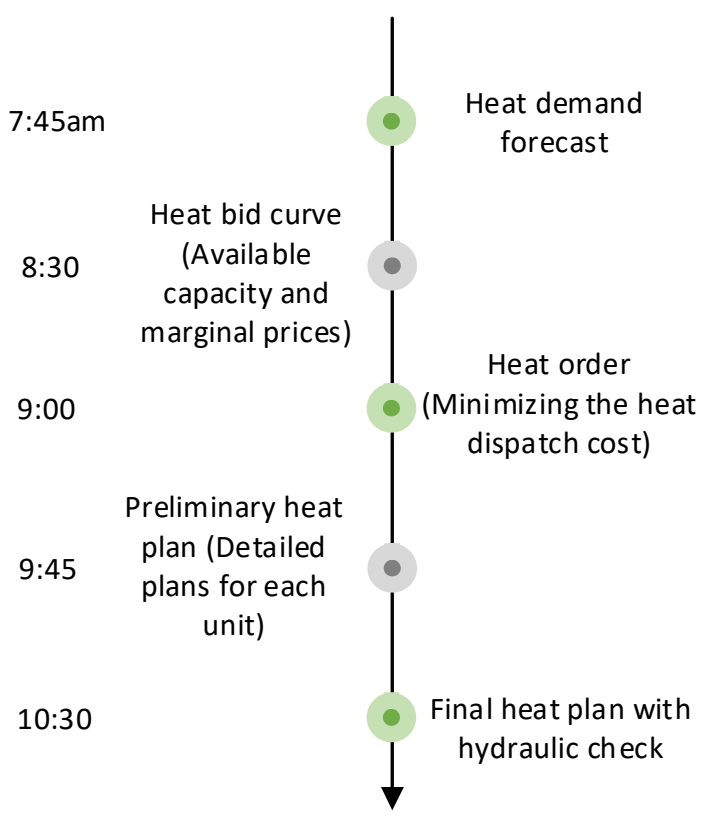

Fig. 9. Procedure for daily heat plans

When the sequential clearing is adopted, the heat production of CHP is determined in the heat-clearing process, extremely limiting the power production of CHP in the EPS. Therefore, the current clearing order for heat and electricity is not suitable for promoting the flexibility to accommodate more renewable energy.

To deal with the challenge in sequential clearing, several studies have explored new market clearing schemes in their modeling for the IEHS to achieve the overall efficiency improvement and fair allocation of heat and power production. Ref. [22] proposes a new market structure to exploit the synergy of two markets. The model still follows the existing market-clearing order of heat and electricity. However, it adopts a stochastic hierarchical optimization model to take account of the flexibility needed in the EPS. At the upper-level, the heat operator minimizes the cost of heat, while accounting for the flexibility requirements of the followers in the day-ahead electricity market. The day-ahead electricity market clearing under different scenarios is 
647 explicitly modeled in the lower level optimization problems. Ref. [97] develops a two-stage

648 decision-making model to maximize the profits in the day-ahead heat market and electricity 649 markets. Ref. [45] proposes a new heat-power market scheme in which the heat market and 650 power market are treated equally and cleared by different operators. Different from the 651 traditional constant heat prices, it determines the heat prices by optimal heat flow.

652 Similar to the decentralized dispatch without market setup, the decentralized clearing is also 653 researched to protect the information of each participant. In Ref. [27], a market-driven model is 654 proposed to achieve the decentralized operation of the EPS and DHS. In the proposed joint 655 market clearing scheme, the EPS operator clears the distribution power market given the 656 demands from the DHS. Simultaneously, the DHS operator determines the optimal heat 657 allocation strategy with given nodal electricity prices. The coordinated optimization between 658 the two subsystems turns into a Nash-type game, and the market equilibrium is the point neither 659 system has an incentive to alter their strategies.

660 In summary, the modeling for the IEHS market clearing is mainly based on the sequential 661 clearing and decentralized clearing to take account of the mutual influence between subsystems. 662 Fig. 10 compares the main difference between sequential clearing and decentralized clearing. 
663
Day-ahead market of heat

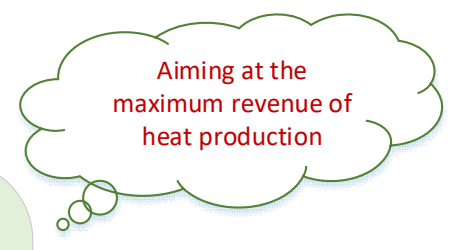

Disclosure of heat plan at 10:00 of the previous day
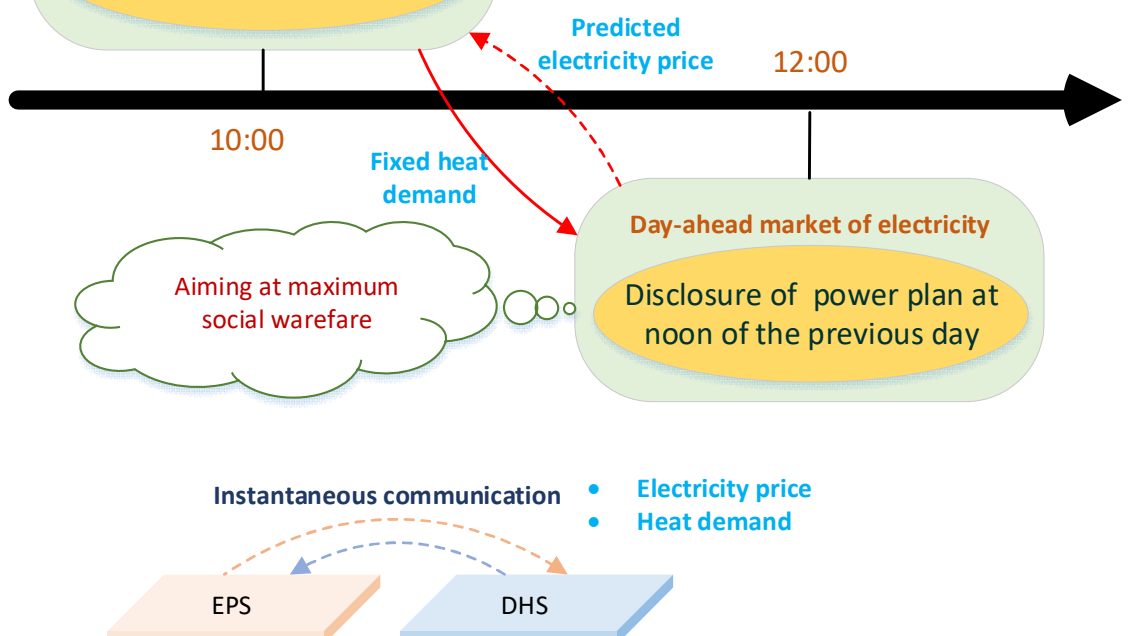

Decentralized

clearing

Decentralized scheme

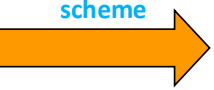

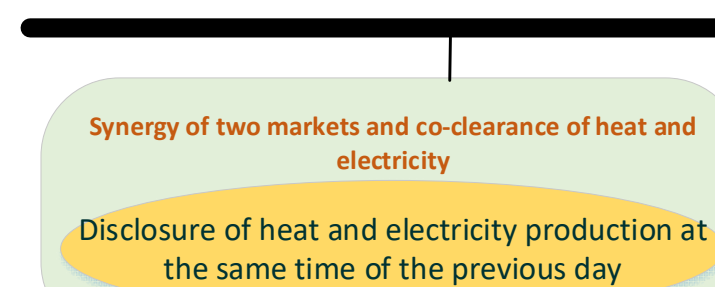
the same time of the previous day

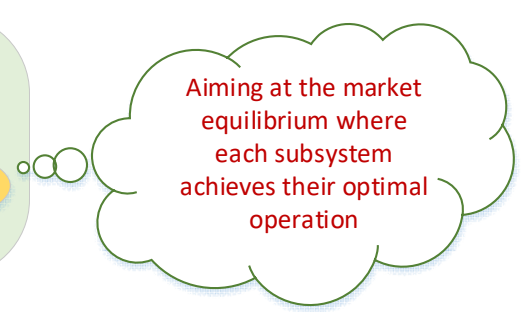

Fig. 10 Comparison of sequential clearing and decentralized clearing

As can be seen from Fig. 10, one of the main differences is the market clearing time. In the sequential clearing, operators make decisions according to predicted or known information. Even though the instantaneous communication is not needed, the predicted information has uncertainty and the known heat information may limit the feasible region of subsequent electricity optimization.

In comparison, in the decentralized clearing, the two subsystems are more interactive, exchanging information iteratively until the equilibrium is reached. With such a scheme, the flexibility needed by the EPS is more easily to be satisfied, and the heat production can be more 
cost-effective according to the actual clearing price. However, instantaneous communication is required due to the simultaneous optimization.

\section{Solution methods for optimal operation of IEHS}

The IEHS model for optimal operation has complex network constraints and system characteristics, which brings a large computational burden and nonlinear and nonconvex properties. Great efforts have been made to seek better decision-making tools for the optimal operation of the IEHS. Existing research on the solution methods of the IEHS for optimal operation is summarized in this section according to the four most common situations, as illustrated in Fig. 11. The following subsections detail these solution methods.

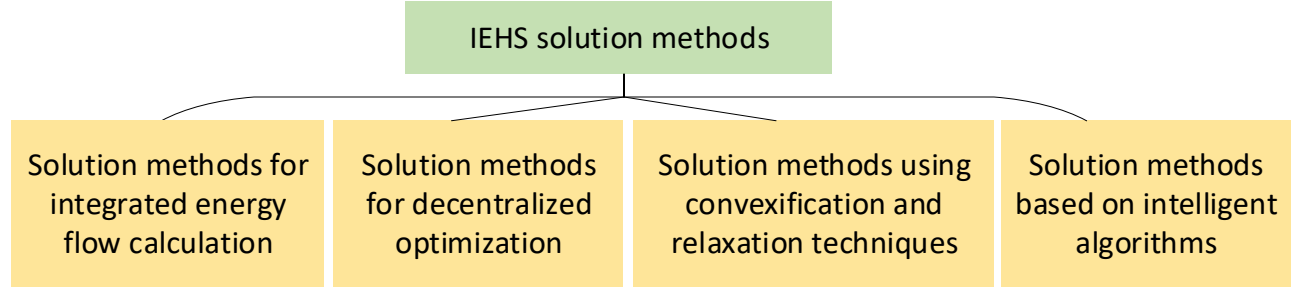

Fig. 11 Solution methods for the optimal operation of the IEHS in four common situations

\subsection{Solution methods for integrated energy flow calculation}

The combined energy flow in the IEHS can be calculated by two kinds of methods, i.e., the integrated and decomposed electrical-hydraulic-thermal methods. The former solves the integrated energy flow as a whole while the latter solves the energy flow in different subsystems sequentially.

The integrated method usually utilizes the Newton-Raphson iteration to solve the energy flow in different subsystems simultaneously. Ref. [70] utilizes the Newton-Raphson iteration to solve the steady-state power flow considering electrical power, natural gas, and district heating. The general iterative form of the Newton-Raphson method for nonlinear equation $\boldsymbol{F}(\boldsymbol{x})=\mathbf{0}$ is as follows [23]:

$$
\boldsymbol{x}^{i+1}=\boldsymbol{x}^{i}-J^{-1} \Delta \boldsymbol{F}(\boldsymbol{x})
$$

where $\boldsymbol{F}(\boldsymbol{x})=\mathbf{0}$ represents all constraints of the IEHS system, $i$ is the index for iterations, $\boldsymbol{x}$ is the vector of variables, $J$ is the Jacobian matrix, which is the first order derivative of $\boldsymbol{F}(\boldsymbol{x})$ 
with respect to $\boldsymbol{x}$, and $\Delta \boldsymbol{F}(\boldsymbol{x})$ is the left hand side of $\boldsymbol{F}(\boldsymbol{x})=\mathbf{0}$.

The integrated method based on Newton-Raphson iterations needs fewer iteration steps. However, it may have the convergence problem if the initial condition is not properly set. Besides, when more complicated constraints are considered, this method may no longer be applicable. For example, when the mass flow direction is considered, the solution in the current iteration will vary significantly if the direction of mass flow in some pipes vary, leading to the difficulty to converge.

Different from the integrated method, the decomposed method calculates each subsystem sequentially, as shown in Fig. 12. The results for one subsystem are the inputs of the other subsystem. Usually, the EPS is solved firstly. Then the DHS is calculated with the fixed electricity as input parameters of coupling components. Besides, when the mass flow rate of the DHS is specified, the DHS can be further decomposed into the independent hydraulic model and thermal model. As the hydraulic process is faster than the thermal process, the hydraulic model is solved before the thermal model.

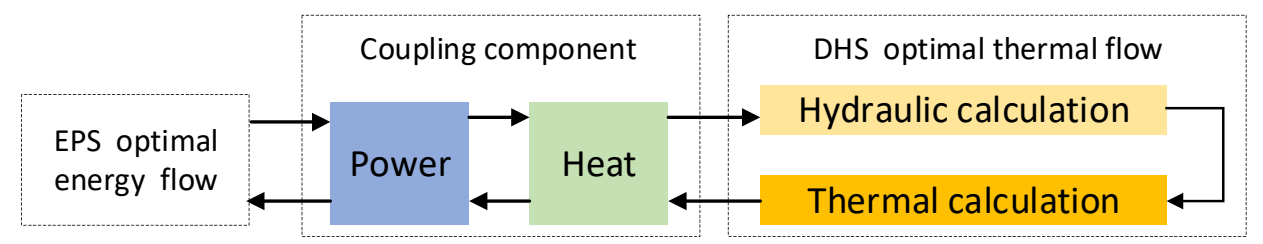

Fig. 12 The iteration procedure for the decomposed method

The decomposed method has better convergence than the Newton Raphson method. Besides, with the decomposition, the energy flow after a disturbance is easy to be analyzed. For example, Ref. [24] utilizes the decomposed method to solve and analyze the energy flow after disturbance. As the EPS and DHS have different dynamic response time, the energy flow in different stages after the disturbance is usually different. In such a situation, the node type of the heat network will change during different stages after the disturbance. With the decomposition, the DHS can change its node type conveniently.

However, the decomposed method may suffer from the calculation burden problem. According to [60], the number of iterations increases with the size of networks for this method. Therefore, it will be a challenge for the decomposed method to guarantee the computation time. 
The decentralized optimization method is used for situations where the EPS and DHS belong to different operators, as stated in subsections 3.2.4 and 3.2.5. The compact form of the formulation in Table 3 for the IEHS is summarized as follows [26]:

$$
\begin{aligned}
\min _{x_{E}, x_{H}} & f_{E}\left(x_{E}\right)+f_{H}\left(x_{H}\right) \\
\text { s.t. } & A_{E} x_{E} \leq b_{E} \\
& A_{H} x_{H} \leq b_{H} \\
& C_{E} x_{E}+D x_{H} \leq h
\end{aligned}
$$

where $x_{E}$ and $x_{H}$ represent the variables of the EPS and DHS, respectively; $A_{E}, A_{H}, C$, and $D_{H}$ are coefficient matrices; $b_{E}, b_{H}$, and $h$ are coefficient vectors; (2.a) and (2.b) are constraints of the EPS and DHS, respectively, and (2.c) is the coupling constraint.

The basic procedure of the decentralized optimization is illustrated in Fig. 13, which can be summarized as follows.

- Step 1: The EPS operator calculates its initial solution based on its own information, then the derived optimal solution is sent to the DHS. The interactive information from the EPS can be the clearing price, power of CHP units, power consumption of HP devices, and so on. The interactive information differs in different decentralized schemes.

- Step 2: The DHS operator calculates its optimal solution with the information from the EPS. Then the interactive information from the DHS is generated, which can be the heat supply from CHP units, power flow on the line connected to the EPS, the feasibility cuts, the optimal cuts, and so on. This information also differs in different decentralized schemes.

- Step 3: With the updated information from the DHS, the EPS operator calculates its optimal solution once again.

- Step 4: If the difference between the optimal solution in the current iteration and the one in the last iteration is within the predefined range, then terminate the procedure and output the optimal solution as the final decision; otherwise, return to Step 2 and repeat the procedure. 
EPS operator

$$
\min _{x_{E}, \eta} f_{E}\left(x_{E}\right)+\eta
$$

s.t. $A_{E} x_{E} \leq b_{E}$

Optimal cut constraints about $\eta$

Feasible cut constraints about interactive information information

Clearing prices, power for heating production,...

Heat demand, heat supply of CHP,...
DHS operator

$$
\min _{x_{H}} f_{H}\left(x_{H}\right)
$$$$
\text { s.t. } A_{H} x_{H} \leq b_{H}
$$

Constraints for

interactive information

Fig. 13 The solving procedure for decentralized method

The solution methods for decentralized optimization have been adopted in several work in the field of the IEHS. The main difference between them lies in how to decompose the original problem in (4) and how to choose the exchanged information.

In Ref. [18], the CHP units, non-CHP thermal units, and wind farms belong to the EPS operator, while the heating boilers belong to the DHS operator. The heat balance constraints for nodes which has CHP and heat boilers are chosen as the coupling constraints. The Benders decomposition has been utilized to generate feasible cuts or optimal cuts of the interactive information. The convergence criterion is whether the difference of optimal solutions of the EPS between two consecutive iterations is less than the predefined tolerance.

Different from Ref. [26], the CHP units in Ref. [72] belong to the DHS operator. Therefore, the EPS and DHS will influence the power flow of transmission lines together, and the power flow constraint on the tie-lines connecting the EPS and DHS is chosen as the coupling constraint between two subsystems. The optimality condition decomposition (OCD) [111,112] method is adopted to generate interactive information. Compared with Ref. [26], less interactive information is needed for the OCD method, and only the Lagrange multipliers of the tie-line constraints and the phase angles of the two ends of the tie-line are needed. Therefore, such a decomposition scheme is more beneficial to protect the privacy and achieve a fully distributed solution. However, the authors neglect the condition that a nodal bus contains both the EPS and DHS components. It is necessary to exploit decomposition schemes applicable to more complicated coupling conditions in the future.

The decentralized optimization method in Ref. [27] considers the market setup, and the interactive information from the EPS to the DHS is the local marginal price and the offered heat 
price of CHP units belonging to the EPS; while the information from the DHS to the EPS is the power demands of HPs and electricity bids of CHP units belonging to the DHS.

The alternating direction method of multipliers (ADMM) is an alternative method to construct a solution framework for decentralized optimization. Ref. [13] utilizes the ADMM to solve the decentralized dispatch of the IEHS, in which the balance constraint between the heat generation and heat demand is selected as the relaxed constraint. Ref. [44] utilizes the ADMM to solve the decentralized optimization of multiple IEHSs with wind power uncertainty incorporated. In the proposed method, the multiple IEHSs are coupled by the tie-line power flow constraints, which can be decomposed by the ADMM.

In summary, the aim of the decentralized optimization methods is to keep each sub-system's information private during the decision-making, while achieving the global or near-global optimum of the whole system. As the decision-making for each subsystem can be performed by off-the-shelf solvers, the optimization of each subsystem is not the core of the decentralized optimization. The main concerns of solution methods for decentralized optimization lie in how to design the decomposition scheme and how to update and exchange information, as well as how to design the update strategy.

\subsection{Solution methods using relaxation and convexification techniques}

To make the optimization of the IEHS computationally tractable, a lot of research utilizes relaxation and convexification techniques to approximate the intractable original optimization problem into a linear programming (LP) problem or a mixed-integer linear programming (MILP) problem. These techniques can be used for all the modeling in Section 3.2.

The relaxation and convexification techniques can be classified into multiple categories according to their application scenario, which are detailed in the following subsections.

\section{Convexification of device characteristic}

The CHP units may have a nonconvex feasible region as illustrated in subsection 3.1.4. Ref. [28] divides the original nonconvex feasible region of CHP units into two separate convex regions and introduces binary variables to indicate which region is reached. As there is only one region reached at a time, the sum of two binary variables is set as 1 . This method achieves the convexification of the feasible region by increasing variables and constraints. Ref. [69] utilizes 
Benders decomposition to separate heat and electricity variables, and the nonconvex feasible region can be more accurately represented.

Most references set the COP of HPs as a constant value, and the function of the operational cost of HPs is in a linear form. However, if HPs take the ambient air as their energy sources, their COP will be variable due to the obvious diurnal air temperature variation during a day. The operation cost function of HPs with variable COP is nonlinear, which cannot be solved directly. For the nonlinear cost function of HPs, Ref. [27] firstly proves the convexity of the HPs' feasible operation region and then reformulates the original cost function with a canonical second-order cone constraint and a set of polyhedral constraints, both of which are compatible with secondorder cone program (SOCP) solvers.

\section{Simplification and relaxation for network constraints}

In the EPS, the network constraints are nonlinear when the AC power flow is modeled at the distribution level. The branch flow model [113] has been widely adopted to transform the original optimization problem into a SOCP through two relaxation steps. However, the convex relaxation may not be exact, leading to a difference with the actual optimal solution. To solve this challenge, Ref. [114] proposes cthe sequential SOCP approach to recover the actual optimal solution.

As can be seen from Table 3 in Section 3.1, both the mass flow rate in the hydraulic process and the supply and return temperatures in the thermal process are variables. Three characteristics result in the nonlinearity of the DHS network.

The first one is the pressure drop constraint, in which the pressure drop along the pipe is related to the square of the mass flow rate. Such an equation is nonconvex. In Ref. [29], this constraint is convexified to a conic constraint as,

$$
K \cdot m_{b t} \cdot m_{b t} \leq h_{b t}
$$

where $\leq$ is used to limit the maximum pressure, but not affecting the optimization results.

The second one is the exponential term in the temperature loss constraint, expressed as $T O_{b t}^{\mathrm{S} / \mathrm{R}}-T_{b t}^{\mathrm{Ambient}}=\left(T I_{b t}^{\mathrm{S} / \mathrm{R}}-T_{b t}^{\mathrm{Ambient}}\right) e^{-\frac{\lambda L}{C_{p} m_{b t}^{\mathrm{SR}}}}$. In this equation, since $\lambda L \leq C_{p} m_{b t}^{\mathrm{S} / \mathrm{R}}$, the approximation that $e^{-x} \approx 1-x$ can be utilized [115]. Then the temperature loss constraint can be approximately expressed as, 


$$
\begin{gathered}
\text { Submitted to Elsevier Science } \\
T O_{b t}^{\mathrm{S} / \mathrm{R}}-T_{b t}^{\mathrm{Ambient}}=\left(T I_{b t}^{\mathrm{S} / \mathrm{R}}-T_{b t}^{\mathrm{Ambient}}\right) \frac{\lambda L}{C_{p} m_{b t}^{\mathrm{S} / \mathrm{R}}}
\end{gathered}
$$

Substitute (4) into the heat loss equation $\Delta Q_{b t}^{\mathrm{S} / \mathrm{R}}=C_{p} m_{b t}^{\mathrm{S} / \mathrm{R}}\left(T O_{b t}^{\mathrm{S} / \mathrm{R}}-T_{b t}^{\mathrm{Ambient}}\right)$, (5) can be obtained,

$$
\Delta Q_{b t}^{\mathrm{S} / \mathrm{R}}=\lambda L\left(T I_{b t}^{\mathrm{S} / \mathrm{R}}-T_{b t}^{\mathrm{Ambient}}\right)
$$

The approximation for (4) and (5) has been validated in [116] by experiments in real heat systems, which shows that the difference between the inlet temperature of a pipeline and the ambient temperature is the main reason for the heat loss. This means the heat loss is independent of the mass flow rate and adjusting the inlet temperature is the sole measure to change losses. The approximation for the heat loss constraint has been adopted by several work.

Besides, on the basis of (5), Ref. [29] further simplifies the heat loss of a pipeline, estimated by,

$$
\Delta Q_{b t}^{\mathrm{S} / \mathrm{R}}=\lambda L\left(T_{r e f}^{\mathrm{S} / \mathrm{R}}-T_{b t}^{\mathrm{Ambient}}\right)
$$

where $T_{r e f}^{\mathrm{S} / \mathrm{R}}$ is the reference temperature of a supply/return network, leading to the heat loss to be a constant. This simplification is established based on the fact that the supply/return temperature is close to the given temperature level.

The third one is the product of the mass flow rate and supply/return temperatures. Two types of methods have been developed to solve this problem.

The first type of method treats one of the variables as a constant according to the adopted heat regulation mode. The quality regulation mode and quantity regulation mode are the two main techniques to satisfy the heat demand. The former treats the mass flow rate as a constant while the supply temperature is variable. In contrast, the latter considers a variable mass flow rate and a constant supply temperature. With one set of variables fixed, the constraints are linear. Ref. [72] adopts the quality regulation mode to linearize the constraints. Ref. [74] utilizes the quantity regulation mode to linearize the constraints.

The second type of methods utilizes the convexification technique to cope with the nonlinear terms, in which both mass flow rate and temperature are variables. Ref. [29] reformulates the nonlinear terms into SOCP forms. 
In summary, the simplification and convexification of the nonlinear terms should rely on the specific characteristics of the network constraint. These techniques should be carefully designed to maintain the accuracy of the approximation.

\section{Reformulation for nonlinear terms}

The modeling of the IEHS for RO is computationally intractable due to the nonlinear terms. The affinely adjustable RO needs to transform a constraint with uncertainty variables into a deterministic one through the robust counterpart of the single inequality constraint [97]. The two-stage adaptive RO is more complicated in calculation due to the min-max-min operator.

The abstract form of the two-stage adaptive RO problem is as follows,

$$
\left\{\begin{array}{l}
\min _{\boldsymbol{X}, \boldsymbol{Z}, \boldsymbol{Y}}\left\{f(\boldsymbol{X})+\max _{\boldsymbol{Z}} \min _{\boldsymbol{Y}} \boldsymbol{c}^{\mathrm{T}} \boldsymbol{Y}\right\} \\
\text { s.t. } \boldsymbol{g}(\boldsymbol{X}) \leq \boldsymbol{b} \\
\boldsymbol{H} \boldsymbol{X}+\boldsymbol{N} \boldsymbol{Y} \leq \boldsymbol{h}-\left(\boldsymbol{E}_{1} \boldsymbol{Z}^{+}-\boldsymbol{E}_{2} \boldsymbol{Z}^{-}\right)
\end{array}\right.
$$

where $\boldsymbol{X}$ is the decision vector of the first stage, and $\boldsymbol{Y}$ is the deterministic decision vector of the second stage, and $\boldsymbol{Z}=\left\{Z^{+}, Z^{-}\right\}$is the uncertainty decision vector of the second stage. $\boldsymbol{H}$ and $\boldsymbol{N}$ are the coefficient matrices; $\boldsymbol{b}, \boldsymbol{c}$, and $\boldsymbol{h}$ are the coefficient vectors.

Solving (7) has two difficulties: the first is that the min-max-min optimization problem cannot be solved directly; the other is the max-min problem in the second stage is nonlinear and nonconvex.

For the first difficulty, the decomposition method such as Benders and C\&CG [19] are usually utilized to decompose the original problem into a master problem (MAP) and a sub-problem (SUP), as shown in (8) and (9), respectively.

$$
\begin{aligned}
& \text { MAP : }\left\{\begin{array}{l}
\min _{\boldsymbol{X}, \eta, \boldsymbol{Y}_{l}}\{f(\boldsymbol{X})+\eta\} \\
\text { s.t. } \boldsymbol{g}(\boldsymbol{X}) \leq \boldsymbol{b} \\
\eta \geq \boldsymbol{c}^{\mathrm{T}} \boldsymbol{Y}_{l}, \forall l \leq k \\
\boldsymbol{H} \boldsymbol{X}+\boldsymbol{N} \boldsymbol{Y}_{\boldsymbol{l}} \leq \boldsymbol{h}-\left(\boldsymbol{E}_{1} \boldsymbol{Z}_{l}^{+*}-\boldsymbol{E}_{2} \boldsymbol{Z}_{l}^{+*}\right), \forall l \leq k \text { (c) }
\end{array}\right. \\
& \text { SUP : }\left\{\begin{array}{l}
R\left(\boldsymbol{X}^{*}\right)=\max _{\boldsymbol{Z}} \min _{\boldsymbol{Y}} \boldsymbol{c}^{\mathrm{T}} \boldsymbol{Y} \\
\text { s.t. } \quad \boldsymbol{H} \boldsymbol{X}^{*}+\boldsymbol{N} \boldsymbol{Y} \leq \boldsymbol{h}-\left(\boldsymbol{E}_{1} \boldsymbol{Z}^{+}-\boldsymbol{E}_{2} \boldsymbol{Z}^{-}\right): \boldsymbol{\pi} \text { (b) }
\end{array}\right.
\end{aligned}
$$

The MAP and SUP are solved iteratively until the gap between the upper and lower bounds is smaller than a pre-defined tolerance. 
The second challenge is to solve the max-min problem. Ref. [117] transforms the max-min problem into equivalent KKT conditions with complementary constraints using the strong dual theory. Then the binary variables are introduced to transform the complementary constraints into mixed-integer constraints which can be solved directly. However, due to the increased binary variables, the solution method based on the KKT conditions usually has a high computational burden. Some other research transforms the max-min problem into its dual optimization problem through the weak duality, as shown in (10).

$$
\left\{\begin{array}{l}
\max _{\boldsymbol{Z}^{+}, \boldsymbol{Z}^{\prime, \pi}}-\left(\boldsymbol{h}-\boldsymbol{E}_{1} \boldsymbol{Z}^{+}+\boldsymbol{E}_{2} \boldsymbol{Z}^{-}-\boldsymbol{H} \boldsymbol{X}^{*}\right)^{\mathrm{T}} \boldsymbol{\pi} \\
\text { s.t. } \boldsymbol{c}+\boldsymbol{N}^{\mathrm{T}} \boldsymbol{\pi}=0 ; \boldsymbol{\pi} \geq 0 ; \boldsymbol{Z}^{+}, \boldsymbol{Z}^{-} \in\{0,1\}
\end{array}\right.
$$

However, the equivalent form in (10) still cannot be solved directly due to the bilinear terms $\boldsymbol{Z}^{+\mathrm{T}} \boldsymbol{\pi}$ and $\boldsymbol{Z}^{-\mathrm{T}} \boldsymbol{\pi}$. The big-M method with the global optimum [118] and the outer approximation method with the local optimum [119] have been utilized to cope with the bilinear terms. Ref. [43] proposes a convex hull relaxation method to cope with the nonlinear terms, and validates its better performance than the big-M and outer approximation methods.

Fig. 14 summarizes the above solution methods for the max-min problem.

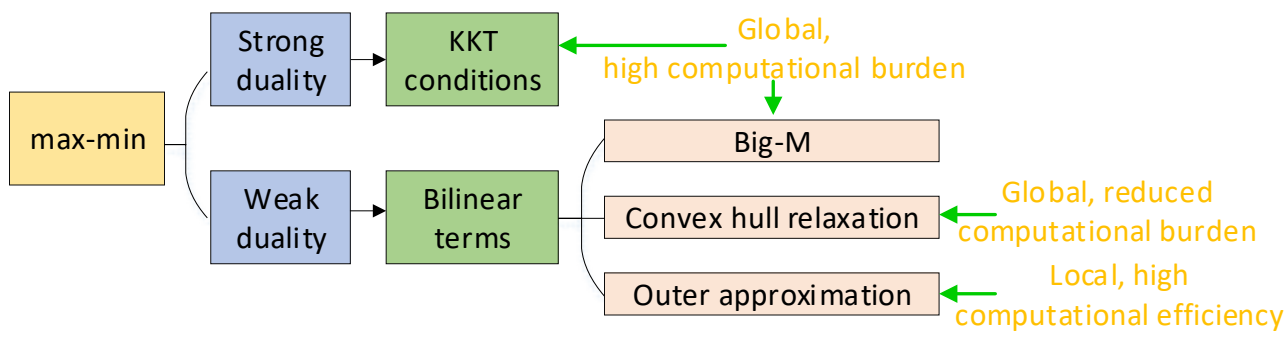

Fig. 14 Summarization for the solution methods of max-min optimization problem

The existing solution methods for the max-min problems are mainly applicable for the EPS scheduling, in which the second-stage constraints are linear. However, the IEHS model based on RO is much complicated due to the nonlinearity of the DHS networks and the different types of uncertainties belonging to different owners. When the nonlinearity is accounted for in the second stage, how to solve the max-min problem with nonlinear constraints will be a new challenge worthy to be exploited in the future. 


\subsection{Intelligent algorithms}

The intelligent algorithms are independent of the types and characteristics of the optimization problem. This type of methods can be utilized to solve the IEHS optimization problem with nonlinear and nonconvex characteristics. The intelligent algorithms, such as gravitational searching [120], group search optimizer [121], and particle swarm optimization [122, 123], have been used to solve the optimal operation of the IEHS. To test the performance of an intelligent algorithm, the quantitative benefits such as the economic efficiency, iteration number, and computation time are usually compared [120]. A more detailed review of the application of intelligent algorithms in the IEHS can be seen in [32].

Even though traditional intelligent algorithms can cope with nonlinear optimization problem, there are two limitations for their application in practice. On the one hand, the intelligent algorithms have low convergence rate [124], failing to satisfy the requirements for online calculation. On the other hand, this type of methods cannot guarantee a stable solution [125]. This is because their performance depends on the initial condition and the values of controllable parameters, which differ in different optimization problems and need a lot of trials to determine the best values.

With the development of artificial intelligence, some research applies the ML methods to the optimization of the IEHS. Ref. [126] utilizes deep reinforcement learning (DRL) to determine the wind power distribution in the EPS and DHS. Ref. [33] proposes an advanced DRL algorithm, i.e., distributed proximal policy optimization (DPPO), to solve the economic dispatch of the IEHS. Ref. [127] proposes a model-free DRL based approach for the real-time dispatch of a residential multi-energy system, which avoids the operational model and parameter forecasting. The application of ML methods in the decision-making of the IEHS is at an early stage, and the relevant research is still limited. This type of methods usually need large amounts of historical data and a lot of time will be used for off-line training. Besides, the parameters in ML methods have a great influence on the solution performance.

\section{Summary and future work}

\subsection{Summary of the IEHS modeling}

In Table 5, the research on IEHS modeling for optimal operation is summarized and compared, with all references listed. 


\begin{tabular}{|c|c|c|c|}
\hline IEHS modeling & & References & Characteristics \\
\hline \multirow{3}{*}{$\begin{array}{l}\text { Modeling for } \\
\text { operation considering } \\
\text { dynamic } \\
\text { characteristics of the } \\
\text { DHS }\end{array}$} & Steady-state model & {$[66],[70],[71]$} & $\begin{array}{l}\text { Neglect the association of the current state with the } \\
\text { previous state for the DHS, e.g. the lack of consideration for } \\
\text { time delays and thermal dynamic characteristic in the } \\
\text { pipelines. }\end{array}$ \\
\hline & $\begin{array}{l}\text { Quasi-dynamic } \\
\text { model }\end{array}$ & $\begin{array}{l}{[11],[13],[19],[26]} \\
{[72],[73],[74]}\end{array}$ & $\begin{array}{l}\text { Consider the thermal process, while keeping the mass flow } \\
\text { rate constant. The current temperature of one node is } \\
\text { influenced by the former temperature of its child node and } \\
\text { the ambient temperature. }\end{array}$ \\
\hline & Dynamic model & [75], [76] & $\begin{array}{l}\text { Consider the dynamics of both the thermal process and } \\
\text { hydraulic process. The temperature and mass flow rates are } \\
\text { both variables. The model is highly nonlinear and } \\
\text { nonconvex. }\end{array}$ \\
\hline \multirow{4}{*}{$\begin{array}{l}\text { Modeling for } \\
\text { operation with } \\
\text { flexibility } \\
\text { improvement }\end{array}$} & IEHS with EBs & {$[62],[72],[78]$} & $\begin{array}{l}\text { The heat production of EBs changes linearly with the power } \\
\text { consumption. }\end{array}$ \\
\hline & IEHS with HPs & $\begin{array}{l}{[27],[79],[80],[81]} \\
{[82],[83],[85],[86]} \\
{[87],[88]}\end{array}$ & $\begin{array}{l}\text { Most HPs have a linear relationship between heat } \\
\text { production and power consumption. Parts of HPs have } \\
\text { variable COP, leading to a nonlinear model. The HPs are } \\
\text { usually equipped with STs. Besides, the HPs can be equipped } \\
\text { at the source side and consumer side. }\end{array}$ \\
\hline & IEHS with STs & [15], [28], [29], [77] & $\begin{array}{l}\text { The water inlet and outlet process, temperature mixing, and } \\
\text { energy storage changes are needed. }\end{array}$ \\
\hline & $\begin{array}{l}\text { IEHS considering } \\
\text { pipeline storage } \\
\text { and building } \\
\text { storage }\end{array}$ & {$[7],[30],[61],[73]$} & $\begin{array}{l}\text { The pipelines and buildings can be seen as virtual flexibility } \\
\text { devices. The pipeline storage is achieved by utilizing the } \\
\text { thermal inertia, but the pipelines can only store a small part } \\
\text { of the heat. } \\
\text { The buildings store and release heat energy by changing the } \\
\text { indoor temperature of heat users. To guarantee the comfort } \\
\text { of users, the indoor temperature should be within a } \\
\text { predefined range. }\end{array}$ \\
\hline \multirow{3}{*}{$\begin{array}{l}\text { Modeling for } \\
\text { operation under } \\
\text { uncertainty }\end{array}$} & $\begin{array}{l}\text { IEHS utilizing SP } \\
\text { technique }\end{array}$ & $\begin{array}{l}{[14],[39],[92],[93]} \\
{[94],[95],[96]}\end{array}$ & $\begin{array}{l}\text { The uncertainties of renewable energy, heat demand, } \\
\text { electricity prices are represented by scenarios. The two- } \\
\text { stage optimization framework is usually adopted. } \\
\text { Considering the correlation of uncertainties can improve } \\
\text { economic efficiency. }\end{array}$ \\
\hline & $\begin{array}{l}\text { IEHS utilizing RO } \\
\text { technique }\end{array}$ & $\begin{array}{l}{[19], \quad[97], \quad[98]} \\
{[103], \quad[104], \quad[105]} \\
{[106]}\end{array}$ & $\begin{array}{l}\text { The uncertainties are represented by box set with budget } \\
\text { constraints. Affinely adjustable RO, adaptive RO, and } \\
\text { distributionally RO are usually adopted for modeling. The } \\
\text { decision based on RO is conservative. }\end{array}$ \\
\hline & $\begin{array}{l}\text { IEHS utilizing the } \\
\text { CCP technique }\end{array}$ & [107] & $\begin{array}{l}\text { Guarantee the security constraints with a probability and } \\
\text { simulates the uncertainties with scenarios. }\end{array}$ \\
\hline \multirow{2}{*}{$\begin{array}{l}\text { Modeling for joint } \\
\text { dispatch of the EPS } \\
\text { and DHS }\end{array}$} & $\begin{array}{l}\text { The centralized } \\
\text { dispatch }\end{array}$ & [108] & $\begin{array}{l}\text { The EPS and DHS are modeled together and the optimal } \\
\text { operation of these two subsystems are determined } \\
\text { simultaneously by the single dispatching center. It is mainly } \\
\text { suitable for a small microgrid managed by a single control } \\
\text { center. }\end{array}$ \\
\hline & $\begin{array}{l}\text { The decentralized } \\
\text { dispatch }\end{array}$ & [76] & $\begin{array}{l}\text { The EPS and DHS belong to different system operators, and } \\
\text { the interactive scheme modeling for information exchange } \\
\text { is needed in the modeling. }\end{array}$ \\
\hline \multirow{2}{*}{$\begin{array}{l}\text { Modeling for joint } \\
\text { market clearing of } \\
\text { the EPS and DHS }\end{array}$} & $\begin{array}{l}\text { The sequential } \\
\text { market clearing }\end{array}$ & {$[22],[45],[97]$} & $\begin{array}{l}\text { The sequential clearing of the EPS and DHS conforms to the } \\
\text { current market policy, with the clearing of the DHS prior to } \\
\text { the EPS. However, the flexibility of the DHS cannot be fully } \\
\text { utilized and the decision is not global optimal. }\end{array}$ \\
\hline & $\begin{array}{l}\text { The decentralized } \\
\text { market clearing }\end{array}$ & [27] & $\begin{array}{l}\text { The decentralized market clearing is more interactive, which } \\
\text { is beneficial to promote flexibility and save cost, but } \\
\text { interactive scheme design is needed in the modeling. }\end{array}$ \\
\hline
\end{tabular}


Table 6 summarizes and compares the solution methods for different situations and using different techniques, with all references listed.

Table 6 Comparison of solution methods for optimal operation of IEHS in different situations

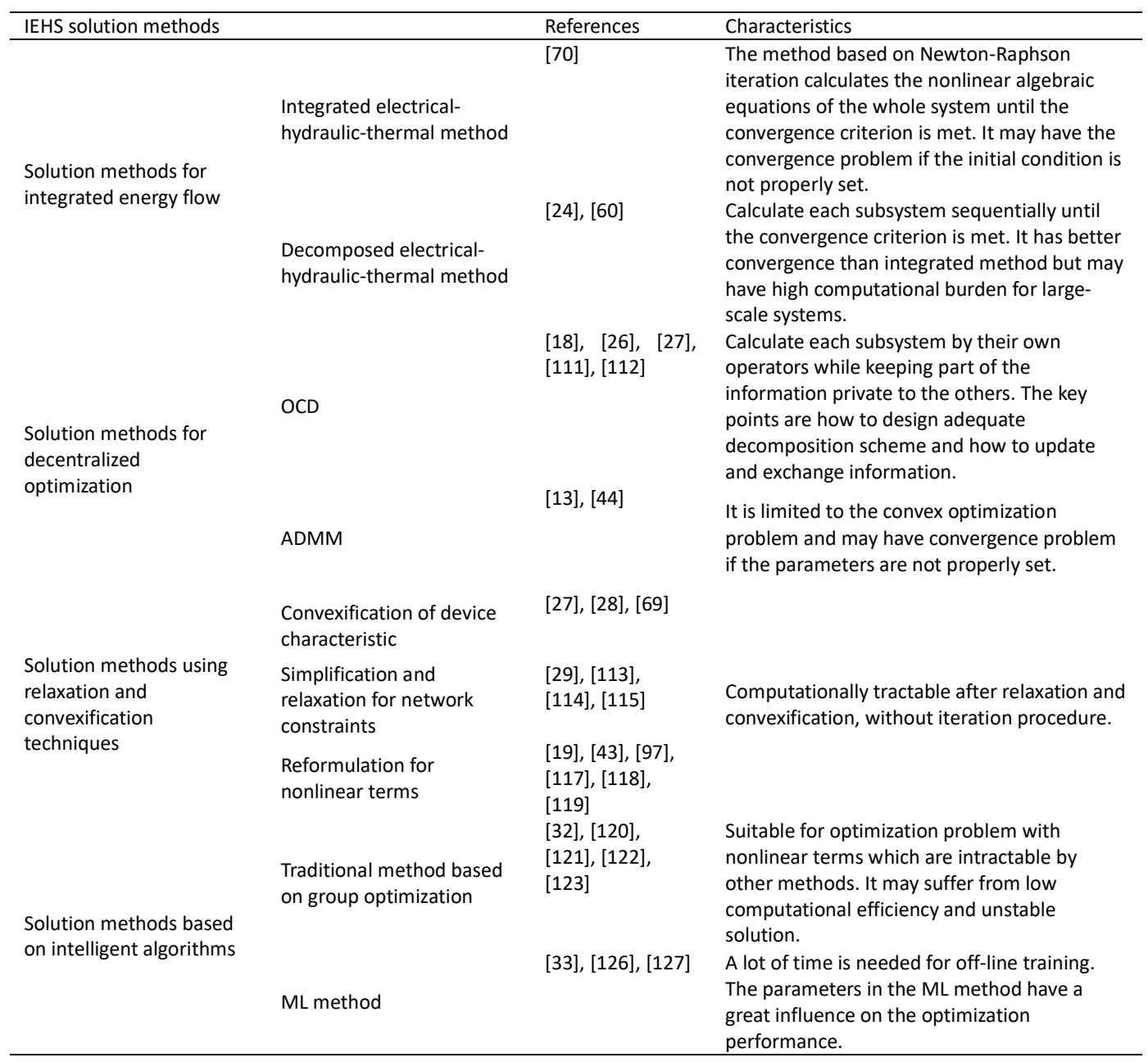

\subsection{Future work}

For the IEHS modeling and solution methods for optimal operation, the following aspects can be researched in the future work.

In the operation considering dynamic characteristics of the DHS, if the DHS could be modeled in a linear form or an easily tractable form for the dynamic characteristics, it will be more preferred. In addition, it is worthwhile to exploit a reasonable time resolution for the joint dispatch of the EPS and DHS in the modeling to properly coordinate the difference in dynamic 
$42 \quad$ Submitted to Elsevier Science

characteristics. In the operation with flexibility improvement, the modeling for schemes promoting the participation of flexible devices can be explored to further increase the flexibility of the system. Flexibility devices can also be made to achieve economic efficiency by providing ancillary services. For example, during periods of high availability of renewable energy, the HPs and EBs can generate more heat to gain benefits from low electricity prices. In the operation under uncertainty, the scenario set for SP and uncertainty set for RO considering the correlations of different uncertainties can be researched to improve the economic efficiency. In the joint dispatch and market clearing of the EPS and DHS, designing reasonable interactive mechanisms between subsystems to harvest more flexibility will be a trend for a fully renewable-based energy system.

In solution methods for integrated energy flow calculation, how to improve the convergence performance of the integrated method and how to reduce the computational burden of the decomposed method can be further explored. In solution methods for decentralized optimization, decomposition scheme suitable for more complex coupling constraints need to be exploited, especially when uncertainties are incorporated in the interactive information. The convergence rate of the ADMM method can be improved by accelerating techniques. In solution methods using relaxation and convexification techniques, the nonlinear terms or nonconvex optimization can be reformulated by using proper simplification or state-of-the-art equivalent transformation in mathematics. In solution methods based on the intelligent algorithm, the ML method is still at an early stage and shall be further studied.

\section{Conclusions}

The IEHS modeling and solution methods are essential for the optimal operation of the IEHS. This paper reviews the IEHS modeling from five aspects of the operation, including the dynamic characteristics of the DHS, operational flexibility improvement, operation under uncertainty, joint dispatch of the EPS and DHS, and joint market clearing of the EPS and DHS. The solution methods are reviewed in four groups, including methods for integrated energy flow calculation, methods for decentralized optimization, methods using relaxation and convexification techniques, and methods based on intelligent algorithms. 
In the modeling for the operation considering dynamic characteristics of the DHS, the quasidynamic model is the most widely researched, which can strike a balance between the tractability and accuracy. The dynamic model will also be encouraged if it can be formulated in a tractable form.

In the modeling for the operation with flexibility improvement, different flexible devices have different operational characteristics and interaction scheme with other parts of the IEHS, leading different constraints for the device and system.

In the modeling for the operation under uncertainty, the uncertainties mainly come from RES, electricity prices, power loads, and heat demand. The modeling using SP usually utilizes a scenario set and two-stage optimization framework to represent and balance uncertainty while the modeling using RO gives priority to the security but suffers from conservativeness. The spatial and temporal correlation of uncertainties can be further exploited to improve the economic efficiency.

In the modeling for joint dispatch of the EPS and DHS, the centralized dispatch is mainly for small-scale grid while the decentralized dispatch focuses on designing reasonable interactive schemes with private information protected and computation burden reduced.

In the modeling for joint dispatch of the EPS and DHS, the sequential market clearing has limited flexibility due to the clearing order. In comparison, decentralized market clearing is much easier to achieve the equilibrium of the EPS and DHS. However, it needs the synergy of clearing guidelines between two subsystems.

In the solution methods for integrated energy flow calculation, the integrated method solves the nonlinear algebraic equations iteratively. It is sensitive to the initial solution and may have convergence issues. The decomposed method is much easier to be convergent, but it may have a large computation burden for the large-scale system.

The solution methods for decentralized optimization are suitable for situations where the EPS and DHS belong to different operators. The existing decomposition schemes of the EPS and DHS differ greatly in the interactive information and update strategy. The design of the interactive information and their presentation form are the key sections of this type of methods, which depends on the system characteristics and joint dispatch and market clearing schemes of the EPS and DHS. The main challenge for this method is the increased computation burden after some equivalent transformation. 
The solution methods using relaxation and convexification techniques are compatible with the other solution methods, which are widely utilized and can be a basis of other methods. The relaxation and convexification techniques should be properly designed, considering both the mathematical features and physical system characteristics.

The solution methods based on intelligent algorithms may have a high computational burden and unstable solution. The traditional intelligent algorithms can be utilized when the nonlinear terms of the optimization are not easy to be transformed and the requirement of calculation time can be met. The ML method needs a large amount of historical data for training, but it can be model-free. The ML method is at an early stage and can be further exploited.

\section{Acknowledgements}

This work was financially supported in part by the Innovationsfond, Denmark, through the project "Using flexible district heating with heat pumps for integrated electricity and heat dispatch with renewables (HEAT4RES)" (8087-00003A), and in part by the Ministry of Science and Technology, China under the project (2018YFE0106600).

\section{Appendix}

To guarantee the high quality of this review paper, a comprehensive search of peer-reviewed journals, conferences, reports, and websites, were completed on a wide range of keywords, including the integrated electricity and heat system, district heating system, electrical power system, optimal operation, combined heat and power units, heat pumps, electric boilers, storage tanks, uncertainty modeling, stochastic programming, robust optimization, electricity markets, dynamic characteristics, centralized dispatch, decentralized dispatch, decentralized market clearing, sequential heat and electricity market clearing, unit commitment, economic dispatch, Newton-Raphson method, decomposed method, ADMM method, decentralized optimization, relaxation and convexification, intelligent algorithms, and machine learning, etc. Five main databases were searched including Elsevier, Springer, IEEE, DTU Findit, and Google scholar. In addition, the literature review section in the found references was searched for additional articles. 


\section{References}

[1] Pinson P, Mitridati L, Ordoudis C, Ostergaard J. Towards fully renewable energy systems: Experience and trends in Denmark. CSEE J Power Energy Syst 2017;3(1):26-35.

[2] Lund R, Persson U. Mapping of potential heat sources for heat pumps for district heating in Denmark. Energy. 2016;110:129-38.

[3] Kroposki B, Johnson B, Zhang Y, et al. Achieving a 100\% renewable grid: Operating electric power systems with extremely high levels of variable renewable energy. IEEE Power Energy M 2017;15(2):61-73.

[4] Wang J, You S, Zong Y, Træholt C, Dong ZY, Zhou Y. Flexibility of combined heat and power plants: A review of technologies and operation strategies. Appl Energy 2019;252:1-13.

[5] Wu Z, Wu J. Feasibility study of district heating with CHP, Thermal Store and Heat Pump. IET Conf Publ. 2013.

[6] Lund H, Werner S, Wiltshire R, et al. 4th Generation District Heating (4GDH). Integrating smart thermal grids into future sustainable energy systems. Energy. 2014;68:1-11.

[7] Gu W, Wang J, Lu S, Luo Z, Wu C. Optimal operation for integrated energy system considering thermal inertia of district heating network and buildings. Appl Energy. 2017;199:234-46.

[8] Rezaie B, Rosen MA. District heating and cooling: Review of technology and potential enhancements. Appl Energy. 2012;93:2-10.

[9] Li J, Fang J, Zeng Q, Chen Z. Optimal operation of the integrated electrical and heating systems to accommodate the intermittent renewable sources. Appl Energy 2016;167:244-254.

[10] Wang Y, You S, Zhang H, et al. Thermal transient prediction of district heating pipeline: Optimal selection of the time and spatial steps for fast and accurate calculation. Appl Energy 2017;206:900-10.

[11] Qin X, Shen X, Sun H, Guo Q. A quasi-dynamic model and corresponding calculation method for integrated energy system with electricity and heat. Energy Procedia. 2019;158:6413-8.

[12] Jie P, Tian Z, Yuan S, et al. Modeling the dynamic characteristics of a district heating network. Energy 2012;39(1):126-34.

[13] Lu S, Gu W, Zhou S, et al. High resolution modeling and decentralized dispatch of heat and electricity integrated energy system. IEEE Trans Sustain Energy 2019; early access.

[14] Zhang M, Wu Q, Wen J, Pan B, Qi S. Two-stage stochastic optimal operation of integrated electricity and heat system considering reserve of flexible devices and spatial-temporal correlation of wind power. Appl Energy 2020;275:115357.

[15] Chen X, Kang C, O'Malley M, et al. Increasing the flexibility of combined heat and power for wind power integration in china: modeling and implications. IEEE Trans Power Syst 2015;30:1848-57.

[16] Wang C, Liu C, Lin Y, et al. Day-ahead dispatch of integrated electric-heat systems considering weatherparameter-driven residential thermal demands. Energy 2020;203:117847.

[17] Yang X, Zhang Y, He H, et al. Real-time demand side management for a microgrid considering uncertainties. IEEE Trans Smart Grid 2018;10(3):3401-14.

[18] Najafi-Ghalelou A, Nojavan S, Zare K, et al. Robust scheduling of thermal, cooling and electrical hub energy system under market price uncertainty[J]. Appl Therm Eng 2019;149:862-80.

[19] Zhou H, Li Z, Zheng J, Wu Q, Zhang H. Robust scheduling of integrated electricity and heating system hedging heating network uncertainties. IEEE Trans Smart Grid 2019; in press.

[20] Turk A, Wu Q, Zhang M, et al. Day-ahead stochastic scheduling of integrated multi-energy system for flexibility synergy and uncertainty balancing[J]. Energy 2020;196:117130.

[21] Varmelast. https://www.varmelast.dk/en/heating-plans.

[22] Mitridati L, Kazempour J, Pinson P. Heat and electricity market coordination: A scalable complementarity approach. Eur J Oper Res 2020;283(3):1107-23.

[23] Liu X, Mancarella P. Modelling, assessment and Sankey diagrams of integrated electricity-heat-gas networks in multi-vector district energy systems. Appl Energy 2016;167:336-52. 
[24] Pan Z, Guo Q, Sun H. Interactions of district electricity and heating systems considering time-scale characteristics based on quasi-steady multi-energy flow. Appl Energy 2016;167:230-43.

[25] Boyd S, Parikh N, Chu E, et al. Distributed optimization and statistical learning via the alternating direction method of multipliers. Foundations and Trends ${ }^{\circledR}$ in Machine learning 2011;3(1):1-122.

[26] Lin C, Wu W, Zhang B, Sun Y. Decentralized solution for combined heat and power dispatch through benders decomposition. IEEE Trans Sustain Energy 2017;8:1361-72.

[27] Cao Y, Wei W, Wu L, Mei S, Shahidehpour M, Li Z. Decentralized operation of interdependent power distribution network and district heating network: A market-driven approach. IEEE Trans Smart Grid 2019;10: 5374-85.

[28] Alipour M, Mohammadi-Ivatloo B, Zare K. Stochastic scheduling of renewable and CHP-based microgrids. IEEE Trans Ind Informatics 2015;11:1049-58.

[29] Huang S, Tang W, Wu Q, Li C. Network constrained economic dispatch of integrated heat and electricity systems through mixed integer conic programming. Energy 2019;179:464-74.

[30] Li Z, Wu W, Wang J, Zhang B, Zheng T. Transmission-constrained unit commitment considering combined electricity and district heating networks. IEEE Trans Sustain Energy. 2016;7:480-92.

[31] Wang C, Gong Z, Liang Y, et al. Data-driven wind generation admissibility assessment of integrated electricheat systems: a dynamic convex hull based approach. IEEE Trans Smart Grid 2020; early access.

[32] Nazari-Heris M, Mohammadi-Ivatloo B, Gharehpetian GB. A comprehensive review of heuristic optimization algorithms for optimal combined heat and power dispatch from economic and environmental perspectives. Renew Sustain Energy Rev 2018;81:2128-43.

[33] Zhou S, Hu Z, Gu W, et al. Combined heat and power system intelligent economic dispatch: A deep reinforcement learning approach. Int J Elec Power 2020;120:1-12.

[34] Yu J, Shen X, Sun H. Economic dispatch for regional integrated energy system with district heating network under stochastic demand. IEEE Access 2019;7:46659-67.

[35] Zhang R, Jiang T, Li W, Li G, Chen H, Li X. Day-ahead scheduling of integrated electricity and district heating system with an aggregated model of buildings for wind power accommodation. IET Renew Power Gener 2019;13:982-9.

[36] Deng B, Fang J, Hui Q, et al. Optimal scheduling for combined district heating and power systems using subsidy strategies. CSEE J Power Energy Syst 2019;5:399-408.

[37] Wu C, Gu W, Jiang P, Li Z, Cai H, Li B. Combined economic dispatch considering the time-delay of district heating network and multi-regional indoor temperature control. IEEE Trans Sustain Energy 2018;9:118-27.

[38] Rong A, Luh PB. A dynamic regrouping based dynamic programming approach for unit commitment of the transmission-constrained multi-site combined heat and power system. IEEE Trans Power Syst 2017;33:71422.

[39] Mitridati L, Pinson P. Optimal coupling of heat and electricity systems: A stochastic hierarchical approach. Int Conf Probabilistic Methods Appl to Power Syst PMAPS 2016.

[40] Zugno M, Conejo A J. A robust optimization approach to energy and reserve dispatch in electricity markets. Eur J Oper Res 2015;247(2):659-71.

[41] Yang Q, Yang T, Li W. Smart power distribution systems: control, communication, and optimization. Academic Press 2018.

[42] Zhao J, Wang H, Liu Y, et al. Coordinated restoration of transmission and distribution system using decentralized scheme. IEEE Trans Power Syst 2019;34(5):3428-42.

[43] Zhang M, Fang J, Ai X, et al. Feasibility identification and computational efficiency improvement for twostage ruc with multiple wind farms. IEEE Trans Sustain Energy 2020;11(3):1669-78.

[44] Tan J, Wu Q, Wei W, et al. Decentralized robust energy and reserve Co-optimization for multiple integrated electricity and heating systems. Energy 2020;205:118040.

[45] Chen Y, Wei W, Liu F, Sauma EE, Mei S. Energy trading and market equilibrium in integrated heat-power distribution systems. IEEE Trans Smart Grid 2019;10:4080-94. 
[46] Zhou B, Ai X, Fang J, Yao W, Zuo W, Chen Z, et al. Data-adaptive robust unit commitment in the hybrid AC/DC power system. Appl Energy 2019;254:113784.

[47] $\mathrm{Wu} \mathrm{C}$, Jiang P, Gu W, et al. Day-ahead optimal dispatch with CHP and wind turbines based on room temperature control. IEEE International Conference on Power System Technology. IEEE 2016;1-6.

[48] DOE U S. Combined heat and power technology fact sheet series. Reciprocating Engines, 2016.

[49] Majanne Y, Seisdedos L V. Sliding back pressure control in industrial CHP plants. IFAC Proceedings Volumes 2012;45(21):639-44.

[50] Oland C B. Guide to combined heat and power systems for boiler owners and operators[M]. United States. Department of Energy, 2004.

[51] Dimoulkas I, Amelin M, Levihn F. District heating system operation in power systems with high share of wind power. J Mod Power Syst Clean Energy 2017;5(6):850-62.

[52] Zhang N, Lu X, McElroy MB, et al. Reducing curtailment of wind electricity in China by employing electric boilers for heat and pumped hydro for energy storage. Appl Energy 2016;184:987-94.

[53] Chen X, McElroy M B, Kang C. Integrated energy systems for higher wind penetration in china: Formulation, implementation, and impacts. IEEE Trans Power Syst 2017;33(2):1309-19.

[54] Gaigalis V, Skema R, Marcinauskas K, Korsakiene I. A review on Heat Pumps implementation in Lithuania in compliance with the National Energy Strategy and EU policy. Renew Sustain Energy Rev 2016;53:841-58.

[55] Skagestad B, Mildenstein P. District heating and cooling connection handbook. Novem, Netherlands Agency for Energy and the Environment, 2002.

[56] Ommen T, Markussen W B, Elmegaard B. Lowering district heating temperatures-impact to system performance in current and future Danish energy scenarios. Energy 2016;94:273-91.

[57] Tan J, Wu Q, Hu Q, et al. Adaptive robust energy and reserve co-optimization of integrated electricity and heating system considering wind uncertainty. Appl Energy 2020;260:114230.

[58] Liao S, Yao W, Han X, et al. Chronological operation simulation framework for regional power system under high penetration of renewable energy using meteorological data. Appl Energy 2017;203:816-28.

[59] Zhang M, Ai X, Fang J, et al. A systematic approach for the joint dispatch of energy and reserve incorporating demand response. Appl Energy 2018;230:1279-91.

[60] Liu X, Wu J, Jenkins N, Bagdanavicius A. Combined analysis of electricity and heat networks. Appl Energy 2016;162:1238-50.

[61] Li Z, Wu W, Shahidehpour M, Wang J, Zhang B. Combined heat and power dispatch considering pipeline energy storage of district heating network. IEEE Trans Sustain Energy 2016;7(1):12-22.

[62] Nielsen MG, Morales JM, Zugno M, Pedersen TE, Madsen H. Economic valuation of heat pumps and electric boilers in the Danish energy system. Appl Energy 2016;167:189-200.

[63] Davidson MR, Perez-Arriaga JI. Modeling unit commitment in political context: Case of China's partially restructured electricity sector. IEEE Trans Power Syst 2018;33:4889-901.

[64] PJM market. https://www.pjm.com/about-pjm.aspx.

[65] Ding T, Li C, Yang Y, Jiang J, Bie Z, Blaabjerg F. A Two-stage robust optimization for centralized-optimal dispatch of photovoltaic inverters in active distribution networks. IEEE Trans Sustain Energy 2017;8:744-54.

[66] Ghorbani N. Combined heat and power economic dispatch using exchange market algorithm. Int J Electr Power Energy Syst 2016;82:58-66.

[67] Liu M, Wang S, Zhao Y, Tang H, Yan J. Heat-power decoupling technologies for coal-fired CHP plants: Operation flexibility and thermodynamic performance. Energy 2019;188:2-14.

[68] Lahdelma R, Hakonen H. An efficient linear programming algorithm for combined heat and power production. Eur J Oper Res 2003;148:141-51.

[69] Abdolmohammadi HR, Kazemi A. A Benders decomposition approach for a combined heat and power economic dispatch. Energy Convers Manag. 2013;71:21-31.

[70] Shabanpour-Haghighi A, Seifi AR. An integrated steady-state operation assessment of electrical, natural gas, and district heating networks. IEEE Trans Power Syst 2016;31:3636-47. 
1167 [71] Wu Q, Zheng J, Jing Z. Coordinated scheduling of energy resources for distributed DHCs in an integrated

[72] Huang J, Li Z, Wu QH. Coordinated dispatch of electric power and district heating networks: A decentralized solution using optimality condition decomposition. Appl Energy 2017;206:1508-22.

[73] Wang D, Zhi Y, Jia H, Hou K, Zhang S, Du W, et al. Optimal scheduling strategy of district integrated heat and power system with wind power and multiple energy stations considering thermal inertia of buildings under different heating regulation modes. Appl Energy 2019;240:341-58.

[74] Shao C, Ding Y, Wang J, Song Y. Modeling and integration of flexible demand in heat and electricity integrated energy system. IEEE Trans Sustain Energy 2018;9:361-70.

[75] Duquette J, Rowe A, Wild P. Thermal performance of a steady state physical pipe model for simulating district heating grids with variable flow. Appl Energy 2016;178:383-93.

[76] Sartor K, Dewalef P. Experimental validation of heat transport modelling in district heating networks. Energy 2017;137:961-68.

[77] Verda V, Colella F. Primary energy savings through thermal storage in district heating networks. Energy 2011;36: 4278-86.

[78] Lu Z, Li H, Qiao Y. Probabilistic Flexibility Evaluation for Power System Planning Considering Its Association With Renewable Power Curtailment. IEEE Trans Power Syst 2018;33:3285-95.

[79] Fischer D, Madani H. On heat pumps in smart grids: A review. Renew Sustain Energy Rev 2017;70:342-357.

[80] Zhang L, Good N, Mancarella P. Building-to-grid flexibility: Modelling and assessment metrics for residential demand response from heat pump aggregations. Appl Energy 2019; 233:709-23.

[81] Nolting L, Praktiknjo A. Techno-economic analysis of flexible heat pump controls. Appl Energy 2019;238:1417-33.

[82] Biegel B, Andersen P, Pedersen T S, Nielsen K, Stoustrup J, Hanse L. Electricity market optimization of heat pump portfolio. CCA Conf Publ 2013.

[83] Lo Basso G, Nastasi B, Salata F, Golasi I. Energy retrofitting of residential buildings-How to couple Combined Heat and Power (CHP) and Heat Pump (HP) for thermal management and off-design operation. Energy Build 2017;151:293-305.

[84] Pedersen TS, Andersen P, Nielsen KM, Starmose HL, Pedersen PD. Using heat pump energy storages in the power grid. Proc IEEE Int Conf Control Appl 2011;1106-11.

[85] Levihn F. CHP and heat pumps to balance renewable power production: Lessons from the district heating network in Stockholm. Energy 2017;137:670-8.

[86] Mathiesen B V., Lund H. Comparative analyses of seven technologies to facilitate the integration of fluctuating renewable energy sources. IET Renew Power Gener. 2009;3:190-204.

[87] Salpakari J, Lund P. Optimal and rule-based control strategies for energy flexibility in buildings with PV. Appl Energy 2016;161:425-36.

[88] Bernath C, Deac G, Sensfuß F. Influence of heat pumps on renewable electricity integration: Germany in a European context. Energy Strateg Rev 2019;26:1-15.

[89] Zheng Q, Wang J; Liu A. Stochastic optimization for unit commitment-A review. IEEE Trans Power Syst 2015;30:1913-24.

[90] Ben-Tal A, Nemirovski A. Robust convex optimization. Math Oper Res 1998;23:769-805.

[91] Zhang M, Fang J, Ai X, et al. Partition-combine uncertainty set for robust unit commitment. IEEE Trans Power Syst 2020;35(4):3266-69.

[92] Zeng Q, Fang J, Chen Z, Conejo AJ. A two-stage stochastic programming approach for operating multi-energy systems. IEEE Conf Energy Internet Energy Syst Integr EI2 2017; 1-6.

[93] Wang J, Wei Z, Yang B, et al. Two-Stage integrated electricity and heat market clearing with energy stations. IEEE Access 2019;7:44928-38. 
[94] Good N, Karangelos E, Navarro-Espinosa A, Mancarella P. Optimization under uncertainty of thermal storage-based flexible demand response with quantification of residential users' discomfort. IEEE Trans Smart Grid 2015;6:2333-42.

[95] Nicholas G, Efthymios K, Alejandro N-E, Pierluigi M. Probabilistic day-ahead CHP operation scheduling. IEEE Power Energy Soc Gen Meet 2015.

[96] Tang C, Wang Y, Xu J, Sun Y, Zhang B. Efficient scenario generation of multiple renewable power plants considering spatial and temporal correlations. Appl Energy 2018;221:348-57.

[97] Zugno M, Morales JM, Madsen H. Commitment and dispatch of heat and power units via affinely adjustable robust optimization. Comput Oper Res. 2016;75:191-201.

[98] Martinez Cesena EA, Mancarella P. Energy systems integration in smart districts: Robust optimisation of multi-energy flows in integrated electricity, heat and gas networks. IEEE Trans Smart Grid. 2019;10:112231.

[99] Li P, Guan X, Wu J, Zhou X. Modeling Dynamic spatial correlations of geographically distributed wind farms and constructing ellipsoidal uncertainty sets for optimization-based generation scheduling. IEEE Trans Sustain Energy 2015;6:1594-605.

[100] Chassein A, Goerigk M. Min-max regret problems with ellipsoidal uncertainty sets. arXiv:160601180v1. 2016:1-25. http://arxiv.org/abs/1606.01180.

[101] Lorca A, Sun XA. Multistage robust unit commitment with dynamic uncertainty sets and energy storage. IEEE Trans Power Syst 2017;32:1678-88.

[102] Dai C, Wu L, Wu H. A multi-band uncertainty set based robust scuc with spatial and temporal budget constraints. IEEE Trans Power Syst 2016;31:4988-5000.

[103] Duan C, Jiang L, Fang W, Liu J, Liu S. Data-driven distributionally robust energy-reserve-storage dispatch. IEEE Trans Ind Informatics 2018;14:2826-36.

[104] Xiong P, Jirutitijaroen P, Singh C. A Distributionally robust optimization model for unit commitment considering uncertain wind power generation. IEEE Trans Power Syst 2017;32:39-49.

[105] Shui Y, Gao H, Wang L, Wei Z, Liu J. A data-driven distributionally robust coordinated dispatch model for integrated power and heating systems considering wind power uncertainties. Int J Electr Power Energy Syst 2019;104:255-8.

[106] Aghaei J, Agelidis VG, Charwand M, et al. Optimal robust unit commitment of CHP plants in electricity markets using information gap decision theory. IEEE Trans Smart Grid 2017;8:2296-304.

[107] Wei Z, Sun J, Ma Z, et al. Chance-constrained coordinated optimization for urban electricity and heat networks. Csee J Power Energy 2018;4(4):399-407.

[108] Pan Z, Guo Q, Sun H. Feasible region method based integrated heat and electricity dispatch considering building thermal inertia. Appl Energy. 2017;192:395-407.

[109] Nordic electricity market. https:/www.nordpoolgroup.com/.

[110] Cities S, Bertelsen N, Petersen U R, et al. Thermal energy storage in greater copenhagen. 2017.

[111] Rabiee A, Mohammadi-Ivatloo B, Moradi-Dalvand M. Fast dynamic economic power dispatch problems solution via optimality condition decomposition. IEEE Trans Power Syst 2014;29:982-3.

[112] Ahmadi-Khatir A, Conejo AJ, Cherkaoui R. Multi-area energy and reserve dispatch under wind uncertainty and equipment failures. IEEE Trans Power Syst 2013;28:4373-83.

[113] Farivar M, Low SH. Branch flow model: Relaxations and convexification. IEEE Trans Power Syst 2013;28:2554-64.

[114] Wei W, Wang J, Li N, Mei S. Optimal power flow of radial networks and its variations: a sequential convex optimization approach. IEEE Trans Smart Grid 2017;8:2974-87.

[115] Li R, Wei W, Mei S, Hu Q, Wu Q. Participation of an Energy Hub in Electricity and Heat Distribution Markets: An MPEC Approach. IEEE Trans Smart Grid 2019;10:3641-53.

[116] Heijde B, Aertgeerts A, Helsen L. Modelling steady-state thermal behaviour of double thermal network pipes. Int J Therm Sci 2017;117:316-27. 
1261 [117] Zeng B, Zhao L. Solving two-stage robust optimization problems using a column-and- constraint generation method. Oper Res Lett. 2013;41:457-61.

[118] Jiang R, Wang J, Guan Y. Robust unit commitment with wind power and pumped storage hydro. IEEE Trans Power Syst 2012;27:800-10.

[119] Bertsimas D, Litvinov E, Sun XA, Zhao J, Zheng T. Adaptive robust optimization for the security constrained unit commitment problem. IEEE Trans Power Syst 2013;28:52-63.

[120] Beigvand SD, Abdi H, La Scala M. Combined heat and power economic dispatch problem using gravitational search algorithm. Electr Power Syst Res 2016;133:160-72.

[121] Jiang XS, Jing ZX, Li YZ, Wu QH, Tang WH. Modelling and operation optimization of an integrated energy based direct district water-heating system. Energy 2014;64:375-88.

[122] Mohammadi-Ivatloo B, Moradi-Dalvand M, Rabiee A. Combined heat and power economic dispatch problem solution using particle swarm optimization with time varying acceleration coefficients. Electr Power Syst Res 2013;95:9-18.

[123] Anand H, Narang N, Dhillon JS. Multi-objective combined heat and power unit commitment using particle swarm optimization. Energy 2019;172:794-807.

[124] Sayed G I, Hassanien A E, Azar A T. Feature selection via a novel chaotic crow search algorithm. Neural Comput Appl 2019;31(1):171-88.

[125] Hu Z, Zhang M, Wang X, et al. Bi-level robust dynamic economic emission dispatch considering wind power uncertainty. Electr Pow Syst Res 2016;135:35-47.

[126] Zhang B, Hu W, Cao D, et al. Deep reinforcement learning-based approach for optimizing energy conversion in integrated electrical and heating system with renewable energy. Energ Convers Manage 2019;202:112199.

[127] Ye Y, Qiu D, Wu X, et al. Model-free real-time autonomous control for a residential multi-energy system using deep reinforcement learning. IEEE Trans Smart Grid 2020; early access. 